ARTICLE

https://doi.org/10.1038/s41467-019-13060-1

\title{
RUNX1 maintains the identity of the fetal ovary through an interplay with FOXL2
}

Barbara Nicol [1 ${ }^{1}$, Sara A. Grimm², Frédéric Chalmel ${ }^{3}$, Estelle Lecluze ${ }^{3}$, Maëlle Pannetier ${ }^{4}$, Eric Pailhoux ${ }^{4}$, Elodie Dupin-De-Beyssat ${ }^{5}$, Yann Guiguen (1) ${ }^{5}$, Blanche Capel ${ }^{6} \&$ Humphrey H.-C. Yao (D) ${ }^{1 *}$

Sex determination of the gonads begins with fate specification of gonadal supporting cells into either ovarian pre-granulosa cells or testicular Sertoli cells. This fate specification hinges on a balance of transcriptional control. Here we report that expression of the transcription factor RUNX1 is enriched in the fetal ovary in rainbow trout, turtle, mouse, goat, and human. In the mouse, RUNX1 marks the supporting cell lineage and becomes pre-granulosa cellspecific as the gonads differentiate. RUNX1 plays complementary/redundant roles with FOXL2 to maintain fetal granulosa cell identity and combined loss of RUNX1 and FOXL2 results in masculinization of fetal ovaries. At the chromatin level, RUNX1 occupancy overlaps partially with FOXL2 occupancy in the fetal ovary, suggesting that RUNX1 and FOXL2 target common sets of genes. These findings identify RUNX1, with an ovary-biased expression pattern conserved across species, as a regulator in securing the identity of ovarian-supporting cells and the ovary.

\footnotetext{
${ }^{1}$ Reproductive and Developmental Biology Laboratory, National Institute of Environmental Health Sciences, Research Triangle Park, Durham, North Carolina 27709, USA. ${ }^{2}$ Integrative Bioinformatics Support Group, National Institute of Environmental Health Sciences, Research Triangle Park, Durham, North Carolina 27709, USA. ${ }^{3}$ Univ Rennes, Inserm, EHESP, Irset (Institut de recherche en santé, environnement et travail) - UMR_S1085, F-35000 Rennes, France. ${ }^{4}$ UMR BDR, INRA, ENVA, Université Paris Saclay, 78350 Jouy-en-Josas, France. ${ }^{5}$ INRA, UR1037 Fish Physiology and Genomics, F-35000 Rennes, France.

${ }^{6}$ Department of Cell Biology, Duke University Medical Center, Durham, North Carolina 27710, USA. *email: humphrey.yao@nih.gov
} 
A critical step that shapes the reproductive identity of the embryo is the sexual differentiation of the bipotential gonads. Supporting cells in the fetal gonads are the first cell population to differentiate and dictate the fate of the gonads. As a consequence, defects in supporting cell differentiation have dire consequences on reproductive outcomes of the individual, from sex reversal to infertility. Supporting cells differentiate into either Sertoli cells, which drive testis development, or pregranulosa cells, which control ovarian development. It has become clear that supporting cell differentiation and maintenance of their commitment requires a coordinated action of multiple factors that play either complementary, redundant, and even antagonistic roles ${ }^{1}$. For instance, fate decision and maintenance of ovarian identity relies mainly on two conserved elements: the WNT4/RSPO1/ $\beta$-catenin pathway ${ }^{2-5}$ and the transcription factor FOXL $2^{6-8}$. These two elements synergistically promote expression of pro-ovarian genes and, at the same time, antagonize key pro-testis factors such as SOX9 and DMRT1. However, the combined loss of these two key pro-ovarian signaling only results in an incomplete inactivation of ovarian differentiation, suggesting that additional pro-ovarian factors are at play during gonadal differentiation ${ }^{9,10}$. Factors involved in gonad differentiation are generally conserved in vertebrates and even invertebrates, although their position in the hierarchy of the molecular cascade may change during evolution ${ }^{11}$. For instance, the proovarian transcription factor FOXL2 is important for ovarian differentiation/function in human ${ }^{12}$, goat ${ }^{13}$, and fish ${ }^{14,15}$. The pro-testis transcription factor DMRT1 is highly conserved and critical for testis development in worms, fly ${ }^{16}$, fish ${ }^{17,18}$, and mammals ${ }^{19-21}$.

In this study, we set up to investigate the role of transcription factor RUNX1 in the mouse fetal ovary. In Drosophila melanogaster, the RUNX ortholog runt is essential for ovarian determination ${ }^{22,23}$. In the mouse, Runx 1 mRNA is enriched in the fetal ovary based on transcriptomic analyses ${ }^{24}$. The RUNX family arose early in evolution: members have been identified in metazoans from sponge to human, where they play conserved key roles in developmental processes. In vertebrates, RUNX1 acts as a transcription factor critical for cell lineage specification in multiple organs and particularly in cell populations of epithelial origin $^{25}$. We first characterize the expression profile of RUNX1 in the fetal gonads in multiple vertebrate species, from fish to human. We then use knockout (KO) mouse models and genomic approaches to determine the function and molecular action of RUNX1 and its interplay with another conserved ovarian regulator, FOXL2, during supporting cell differentiation in the fetal ovary.

\section{Results}

Runxl expression pattern implies a role in ovary development. The runt gene, critical for ovarian determination in the $\mathrm{fly}^{22}$, has three orthologs in mammals: RUNX1, RUNX2, and RUNX3. Although all three RUNX transcription factors bind the same DNA motif, they are known to have distinct, tissue-specific functions ${ }^{26}$. In the mouse, Runx1 was the only one with a strong expression in the fetal ovary, whereas Runx2 and Runx3 were expressed weakly in the fetal gonads in a non-sexually dimorphic way (Fig. 1a). At the onset of sex determination (Embryonic day 11.5 or E11.5), Runx1 expression was similar in both fetal XY (testis) and XX (ovary) gonads before becoming ovary-specific after E12.5 (Fig. 1b), consistent with observations by others ${ }^{24,27}$. An ovary-enriched expression of Runxl during the window of early gonad differentiation was also observed in other mammals such as human and goat, as well as in species belonging to other classes of vertebrates such as red-eared slider turtle and rainbow trout (Fig. 1c-f), implying an evolutionarily conserved role of RUNX1 in ovary differentiation.

To identify the cell types that express Runx1 in the gonads, we examined a reporter mouse model that produces enhanced green fluorescent protein (EGFP) under the control of Runx1 promoter $^{28}$ (Fig. 2 and Supplementary Fig. 1). Consistent with Runx1 mRNA expression (Fig. 1b), Runx1-EGFP was present in both $\mathrm{XX}$ and $\mathrm{XY}$ gonads at E11.5, then increased in XX gonads and diminished in XY gonads at E12.5 onwards (Fig. 2). At E11.5 in both XX and XY gonads, Runx1-EGFP was present in a subset of SF1+/PECAM - somatic cell population, whereas it was absent in the $\mathrm{SF} 1-/ \mathrm{PECAM}+$ germ cells (Fig. $2 \mathrm{a}-\mathrm{d}$ ). In the XY gonads, these Runx1-EGFP+ somatic cells corresponded to Sertoli cells, as demonstrated by a complete overlap with SRY, the sexdetermining factor that drives Sertoli cell differentiation ${ }^{29}$ (Fig. 2e and Supplementary Fig. 1). At this stage, there is no marker for ovarian-supporting cells that allow us to determine which subset of somatic cells were positive for Runx1-EGFP in XX gonads. However, at E12.5, when the sex of gonads becomes morphologically distinguishable, Runx1-EGFP was specifically detected in the supporting cell lineage of both sexes: strongly in FOXL2+ pre-granulosa cells of XX gonads (Fig. 2g) and weakly in SOX9+ Sertoli cells of XY gonads (Fig. 2f and Supplementary Fig. 1). Runx1-EGFP expression was eventually turned off in the fetal testis, while it was maintained in the ovary (Fig. 2h, i). Throughout fetal development of the ovary, Runx1-EGFP remained in FOXL2+ pre-granulosa cells (Fig. 3). Runx1-EGFP was also detected in the ovarian surface epithelium at E16.5 and birth (arrows in Fig. 3b, c), which gives rise to granulosa cells in the cortex of the ovary ${ }^{30,31}$. Runx1-EGFP was also expressed in somatic cells of the cortical region right underneath the surface epithelium at E16.5 and some of these Runx1-EGFP+ cells presented a weak expression of FOXL2 (Fig. $3 \mathrm{~g}-\mathrm{i}$, arrowheads). In summary, Runx1 marks the supporting cell lineage in the gonads at the onset of sex determination and becomes pre-granulosa cellspecific as gonads differentiate.

XX Runx1 KO and Foxl2 KO share common transcriptomic changes. Its pre-granulosa cell-specific expression suggests that RUNX1, a factor involved in cell lineage determination ${ }^{25}$, could contribute to granulosa cell differentiation and ovarian development. To investigate its role in gonads and avoid early embryonic lethality as a result of global deletion of $\operatorname{Run} x 1^{32,33}$, we generated a conditional KO mouse model in which Runxl was ablated in the SF1+ gonadal somatic cells ${ }^{34}$ (Fig. 4). We characterized the effects of Runx1 inactivation on XX gonad differentiation at E14.5, a stage where morphological differences between ovary and testis are already established. Although Runx1 expression was ablated successfully in XX gonads (Fig. 4d), ovarian morphogenesis appeared normal: E14.5 XX Runx1 KO gonads presented similar size and shape compared with XX control gonads (Supplementary Fig. 2), and differentiation and organization of different gonadal cell populations were similar to control XX gonads (Fig. 4a-c). For instance, XX Runx1 KO-supporting cells expressed the pre-granulosa cell marker FOXL2 but not Sertoli cell markers SOX9 and AMH (Fig. 4a, b). Supporting cells and germ-cell organization was similar to XX control gonads and did not form Laminin-outlined cord structures typical of testis differentiation (Fig. 4b). Similar to supporting cells, no difference was observed in the COUP-TFII+ interstitium organization between XX Runx1 KO and XX control gonads (Fig. 4b). Finally, E14.5 XX Runx1 KO germ cells had initiated meiosis, a typical feature of fetal ovary development, and normal expression of the germ-cell marker $M v h$ was observed (Fig. 4c, d). No significant change was detected for key genes involved in pre-granulosa cell 
a

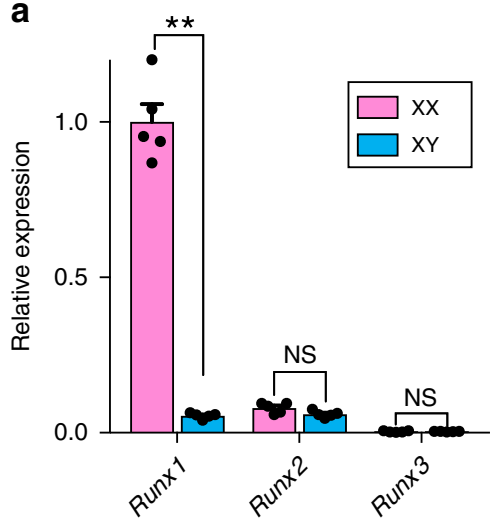

C

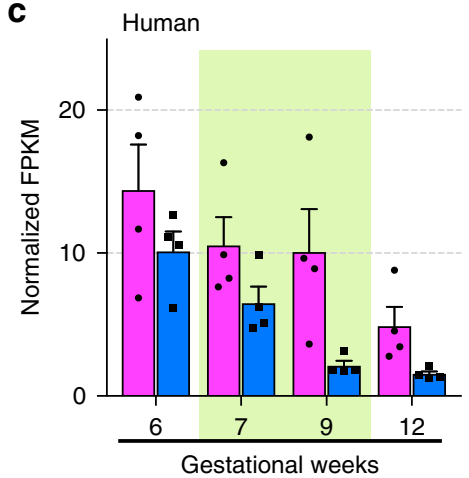

e

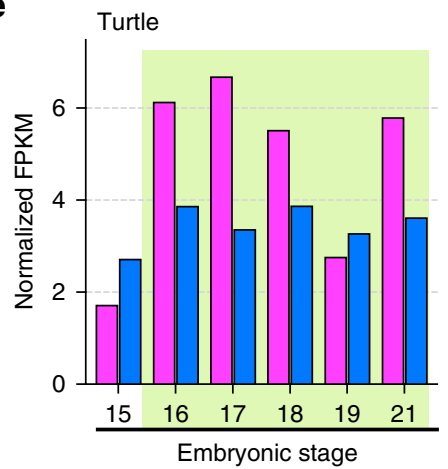

Embryonic stage b

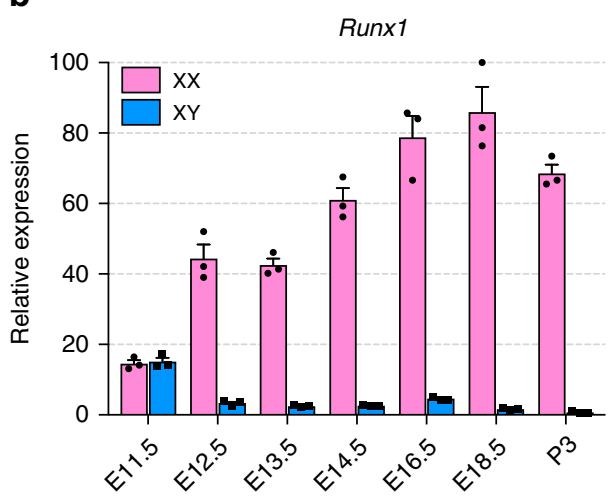

d

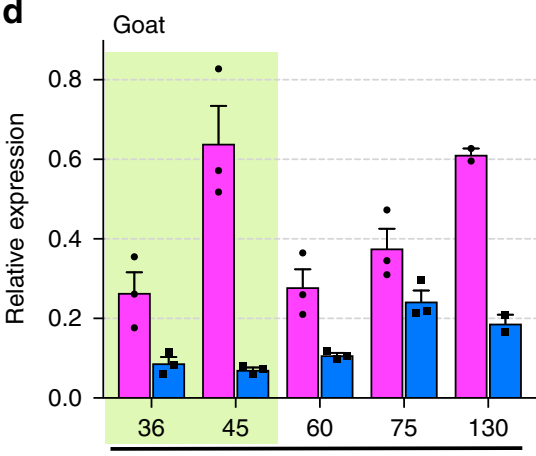

Days post coitum

f

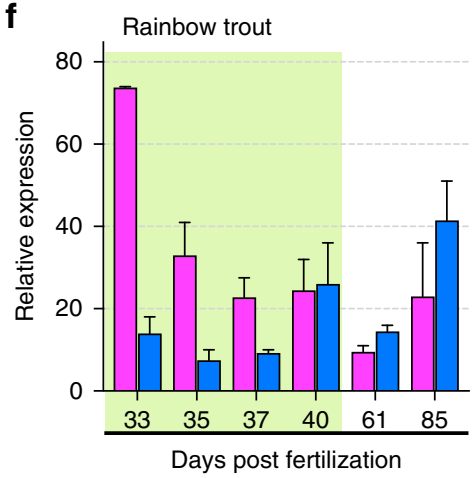

Window of early

gonad differentiation

Fig. 1 RUNX1 expression during gonadal differentiation in various vertebrates. a Expression of Runx1, Runx2, and Runx3 mRNAs in XX and XY gonads of E14.5 mouse embryos $\left(n=5 /\right.$ sex). Values are presented as mean \pm SEM; non-parametric $t$-test, ${ }^{\star \star} p<0.01$; NS, not significant. $\mathbf{b}$ Expression time course of Runx 1 mRNA in mouse $X X$ and $X Y$ gonads during gonadal differentiation $(n=3 /$ stage). Values are presented as mean \pm SEM. $\mathbf{c}-\mathbf{f}$ Time course of $R U N X 1$ mRNA expression in four other vertebrate species, human, goat, red-eared slider turtle, and rainbow trout during gonad differentiation. Values are presented as mean $\pm \mathrm{SEM}$. For the turtle, pink and blue bars represent gonads at female-promoting temperature (FPT) of $31^{\circ} \mathrm{C}$ and at male-promoting temperature (MPT) of $26^{\circ} \mathrm{C}$, respectively ${ }^{64}$. RUNX1 expression was analyzed by RNA-seq in human and red-eared slider turtle $\mathrm{e}^{64}$, and by qPCR in goat and rainbow trout. Green highlighted areas represent the window of early gonadal differentiation. Source data are provided as a Source Data file

differentiation (Foxl2 and Wnt4) or Sertoli cell differentiation (Sox9, Fgf9, Nr5a1, and Amh; Fig. 4d, e). However, pre-granulosa cell marker Fst and fetal Sertoli cell marker desert hedgehog $(D h h)$ were mis-expressed in E14.5 XX Runx1 KO gonads (Fig. 4d, e).

Similarly, at birth, XX Runx1 KO gonads maintained a typical ovarian shape, with FOXL2+ pre-granulosa cells scattered throughout the gonad and TRA98+ germ cells located mostly in the cortex (Fig. 4f). Despite their normal ovarian morphology, newborn XX Runxl KO gonads exhibited an aberrant transcriptomic profile reminiscent of the transcriptome of newborn XX
Foxl2 KO gonads, such as downregulation of pro-ovarian genes that are direct targets of FOXL2 (Fig. 4g and Supplementary Data 1). Foxl2 is involved in ovarian differentiation/maintenance in various vertebrate species. In the mouse, loss of Foxl2 results in normal ovarian morphogenesis at birth, despite aberrant ovarian transcriptome, and eventually leads to masculinization of the ovary postnatally ${ }^{6}$. We found that $41 \%$ of the genes differentially expressed in Runx1 KO were also misregulated in the absence of Foxl2 in newborn XX gonads (Fig. 4g and Supplementary Data 1). However, contrary to Foxl2 KO, loss of Runx1 in the ovary did not result in postnatal sex reversal and the females Runx1 KO 


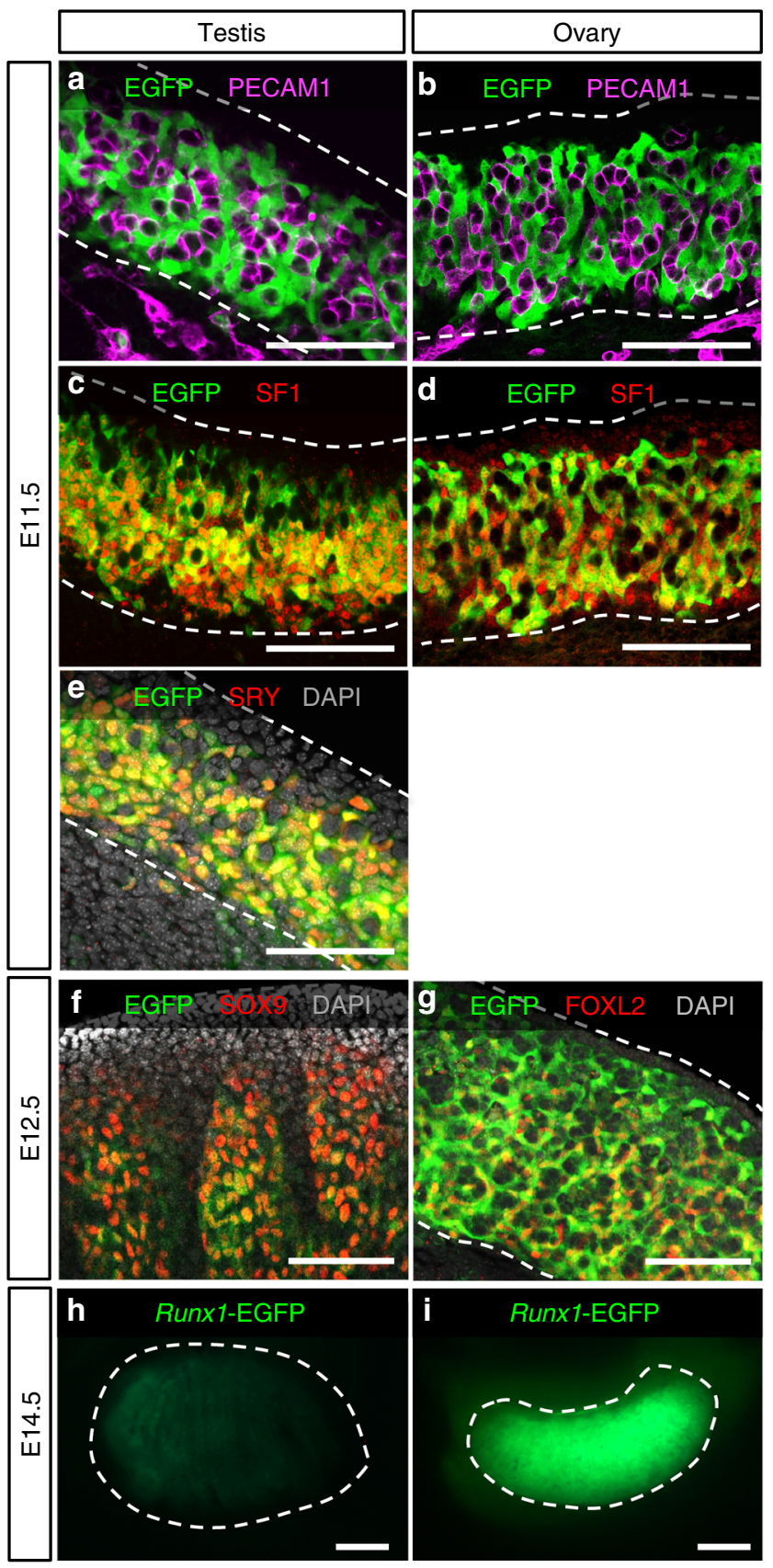

Fig. 2 Run $x 1$ is expressed in the supporting cells during gonad differentiation. $\mathbf{a}-\mathbf{g}$ Whole-mount immunofluorescence of $X Y$ and $X X$ gonads from $\operatorname{Tg}$ (Runx1-EGFP) reporter mice at E11.5 and E12.5. Gonads with endogenous EGFP were co-labeled with markers for germ cells/vasculature (PECAM-1; a, b), somatic cells (SF1; c, d), Sertoli cells in XY gonads (SRY in e and SOX9 in f), and for pre-granulosa cells in XX gonads (FOXL2; $\mathbf{g}$ ). Scale bars: $100 \mu \mathrm{m}$. Single-channel images are provided for $\mathbf{e}, \mathbf{f}$ in Supplementary Fig. 1. h, i Detection of endogenous EGFP in freshly collected E14.5 gonads. Scale bars: $200 \mu \mathrm{m}$. Dotted lines outline the gonads. At least three independent biological replicates were analyzed and the images presented are representative of all replicates

were fertile. One possible explanation for these common transcriptomic changes is that Runxl could be part of the same signaling cascade as Foxl2. However, analysis of Runx1 expression in Foxl2 $\mathrm{KO}$ newborn ovaries did not detect any changes in Runxl expression in the absence of Foxl2 (Fig. 4h). Conversely, Foxl2 expression was not changed in the absence of Runx1. These results indicate that Runxl and Foxl2 regulate common sets of genes but they are themselves regulated independently of each other in the fetal ovary.

Runx1 and Foxl2 double knockout results in partial masculinization of fetal ovaries. The common transcriptomic changes identified in Runx1 and Foxl2 KO newborn ovaries raised the question whether RUNX1 and FOXL2 could play redundant/ complementary roles in supporting cell differentiation. We therefore generated Runx1/Foxl2 double KO mice (referred as $\mathrm{DKO})$ and compared XX gonads differentiation in the absence of Runx1, Foxl2, or both (Fig. 5 and Supplementary Figs. 3 and 4). Abnormal development of XX DKO gonads became apparent around E15.5. At this stage, differentiation of supporting cells into Sertoli cells in the testis or pre-granulosa cells in the ovary has been established. For instance, the transcription factor DMRT1, involved in the maintenance of Sertoli cell identity ${ }^{19}$, is expressed in Sertoli cells but not pre-granulosa cells (Fig. 5a, e). At E15.5, DMRT1 is also present in a few germ cells in both the testis and ovary ${ }^{35}$. Similar to control ovaries, XX gonads lacking either Runx1 or Foxl2 had no DMRT1 proteins in the supporting cells (Fig. 5a-c). However, the combined loss of Runx1 and Foxl2 resulted in aberrant expression of DMRT1 in the supporting cells of XX gonads (Fig. 5d). At birth, a time when XX Foxl2 KO gonads still morphologically resemble ovaries ${ }^{6,7}, \mathrm{XX}$ Runx1/Foxl2 DKO gonads formed structures similar to fetal testis cords in the center, with DMRT1 + cells surrounding clusters of germ cells (Fig. 5i). Such structure was not observed in XX Runx1 KO or Foxl2 KO gonads with the exception that DMRT1 protein started to appear in a few supporting cells in the newborn XX Foxl2 KO gonads, in what appears to be one of the first signs of postnatal masculinization of Foxl2 KO ovaries at the protein level (Fig. 5h). Contrary to DMRT1, SOX9 protein, a key driver of Sertoli cell differentiation $^{36,37}$, was not detected in XX Runx1/Foxl2 DKO newborn gonads (Fig. 6). Our results demonstrate that a combined loss of Runx1/Foxl2 induces partial masculinization of the supporting cells during fetal development of the ovary.

To further characterize the impacts of the combined loss of Runx1/Foxl2 on ovarian differentiation, we compared the transcriptome of newborn XX Runx1/Foxl2 DKO gonads with the transcriptomes of XX control, Runx1, or Foxl2 single KO gonads (Fig. 7 and Supplementary Data 2). The heat map for the 918 differentially expressed genes between XX Runx1/Foxl2 DKO and XX control gonads demonstrated allele-specific impacts: loss of Runx1 resulted in a mild and often nonsignificant effect on these genes, loss of Foxl2 had an intermediate/strong effect, and combined loss of Runx1/Foxl2 yielded the strongest effect (fold change $>1.5 ; p<0.05$ one-way analysis of variance (ANOVA); Fig. 7a and Supplementary Data 3). Gene ontology analysis revealed that the downregulated genes were associated with "ovarian follicle development" and "female gonad development," whereas "male sex determination" was the most significantly enriched process for the upregulated genes (Supplementary Fig. 5). Conforming to the hierarchical clustering (Fig. 7a), comparison of the genes significantly changed in Runx1/Foxl2 single and DKOs suggested that Foxl2 was the main contributor to the transcriptional changes observed in Runx1/Foxl2 DKO (Fig. 7b-g and Supplementary Data 4): 61\% of the genes downregulated in DKO were also downregulated in Foxl2 KO and $43 \%$ of the genes upregulated in DKO were also upregulated in Foxl2 KO. In addition, some genes appeared to be controlled by both Foxl2 and Runx1, and were significantly downregulated or upregulated in all three KOs (Fig. 7b, c). For instance, the genes Fst and Cyp19a, both involved in granulosa cell differentiation/function ${ }^{38,39}$, were downregulated in Runx1 KO, 

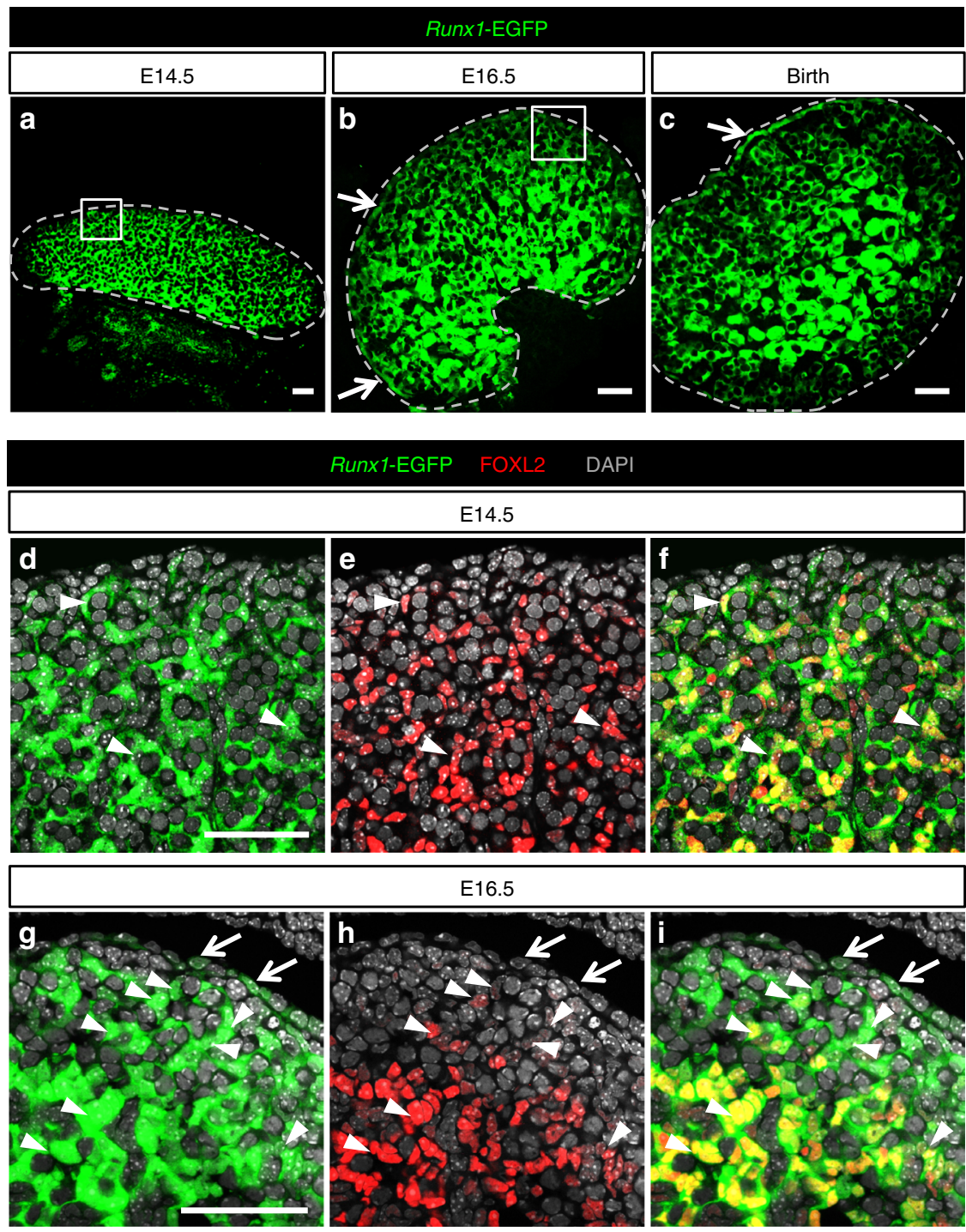

Fig. 3 Runx1 expression is maintained in pre-granulosa cells throughout fetal ovarian development. a-c Immunofluorescence for EGFP on Tg(Runx1-EGFP) ovary sections at E14.5, E16.5, and birth. Scale bars: $50 \mu \mathrm{m}$. d-i Immunofluorescence for EGFP and the pre-granulosa cell marker FOXL2 at E14.5 (d-f) and E16.5 (g-i), corresponding to the white square outlined areas in $\mathbf{a}$ and $\mathbf{b}$, respectively. Runx 1 is expressed in pre-granulosa cells throughout ovarian development and the surface epithelium after E14.5. Dotted lines outline the gonads. Arrows: EGFP + ovarian surface epithelium. Arrowheads: EGFP/ FOXL2 double-positive cells. Scale bars: $50 \mu \mathrm{m}$. At least three independent biological replicates were analyzed and the images presented are representative of all replicates

Foxl2 KO, and more repressed in Runx1/Foxl2 DKO (Fig. 7d). On the other hand, Dhh was upregulated in all three KOs, with the highest expression in the DKO (Fig. 7f). Finally, some genes were significantly changed in Runx1/Foxl2 DKO only, suggesting a cumulative effect of Runx1 and Foxl2 loss. For instance, Foxp1, a gene whose expression is enriched in pre-granulosa cells ${ }^{40}$, was significantly downregulated only in Runx1/Foxl2 DKO (Fig. 7e), whereas Fgf9, a Sertoli gene contributing to testis differentiation $^{41}$, and $P d g f c$ were significantly upregulated only in Runxi/ Foxl2 DKO (Fig. 7g).

In contrast to XX Foxl2 single KO gonads (Fig. 5), in which sex reversal only became apparent postnatally ${ }^{6}$, XX Runx1/Foxl2 DKO gonads exhibited masculinization with visible morphological changes before birth. To determine how the additional loss of Runx1 contributed to the earlier masculinization of Runx1/Foxl2 DKO ovaries, we identified the genes differentially expressed between XX Runx1/Foxl2 DKO and XX Foxl2 KO gonads (Fig. 7h and Supplementary Data 5). Expression of most of these genes was already altered in XX Foxl2 single KO gonads; however, the additional loss of Runxl exacerbated their mis-expression. For instance, the pro-testis gene Dmrt1 and Nr5a1 were significantly upregulated, whereas the pre-granulosa-cell-enriched transcripts Fst and Ryr2 were further downregulated at birth (Fig. 7d, i). On the other hand, the additional loss of Runxl did not cause further upregulation of the Sertoli genes Sox 9 and Amh at birth, suggesting that Runx1 does not contribute to their repression in the ovary (Fig. 7j). Overall, the transcriptomic analyses of Runx1/ Foxl2 single and DKOs revealed that Foxl2 is the main driver of the transcriptomic changes, and that the additional loss of Runx 1 amplifies the mis-expression of genes already altered by the sole loss of Foxl2, leading to the failure to maintain pre-granulosa cell identity in the fetal ovary.

RUNX1 shares genome-wide chromatin occupancy with FOXL2. The masculinization of XX Runx1/Foxl2 DKO fetal gonads and the transcriptomic comparisons of newborn XX Runx1/Foxl2 single and DKO gonads suggest some interplay 

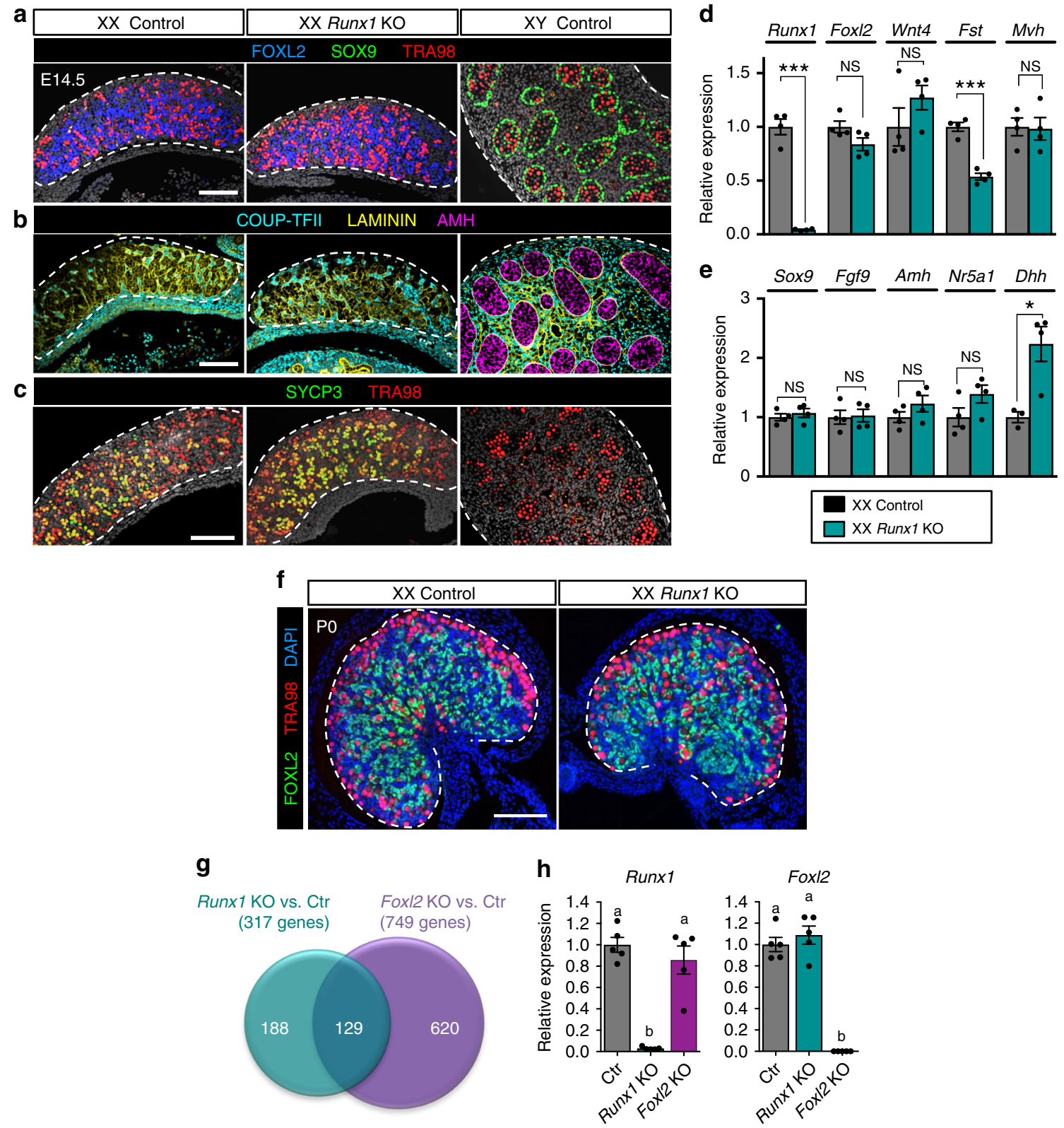

Fig. 4 Inactivation of Runx1 in the ovarian somatic cells has minimal impacts on morphogenesis of the ovary with the exception of transcriptomes. Immunofluorescences for a pre-granulosa cell marker FOXL2, Sertoli cell marker SOX9, and germ-cell marker TRA98; b interstitial cell marker COUP-TFII (encoded by Nr2f2 gene), Laminin, and Sertoli cell marker AMH; c meiotic cell marker SYCP3 and germ-cell marker TRA98 in E14.5 XX control, XX Runx1 $K O$, and $X Y$ control gonads. The gray color represents DAPI nuclear staining. Single-channel images are provided in Supplementary Fig. 2. Expression of $\mathbf{d}$ pre-granulosa or germ-cell-specific genes and e Sertoli or Leydig cell-specific genes in XX control and XX Runx1 KO gonads at E14.5 by quantitative PCR $(n=5)$. Values are presented as mean \pm SEM.; unpaired Student's $t$-test; ${ }^{\star} p<0.05,{ }^{\star \star \star} p<0.001$; NS, not significant. f Immunofluorescence for FOXL2, TRA98, and nuclear counterstain DAPI (blue) in XX control and Runx1 KO gonads at birth (PO). Scale bar: $100 \mu \mathrm{m}$. $\mathbf{g}$ Venn diagram comparing the 317 genes differentially expressed in XX Runx $1 \mathrm{KO}$ vs. XX Control gonads with the 749 genes differentially expressed in XX FoxI2 KO vs. XX Control gonads at birth. Forty-one percent of the genes differentially expressed in Runx1 KO (129/317) were also misregulated in the absence of Foxl2. Genes differentially expressed were identified by microarray $(n=4$ /genotype; fold change $>1.5$, one-way ANOVA $p<0.05)$. $\mathbf{h}$ Quantitative PCR analysis of Runx 1 and Fox $/ 2$ mRNA expression in XX control, Runx1 KO, and Foxl2 KO gonads at birth ( $n=5 /$ genotype). Values are presented as mean \pm SEM. One-way ANOVA, $p<$ 0.05. Bars with different letters $(\mathbf{a}, \mathbf{b})$ are significantly different. For all experiments, controls are wild-type littermates of Runx $1 \mathrm{KO}$ mice. Source data are provided as a Source Data file. For the immunofluorescences, at least three independent biological replicates were analyzed and the images presented are representative of all replicates

between RUNX1 and FOXL2 to control pre-granulosa cell identity. The fact that RUNX1 and FOXL2 are both transcription factors expressed in the pre-granulosa cells (Fig. 3 and Supplementary Fig. 1) raised the question whether this interplay could occur directly at the chromatin level. We have previously identified FOXL2 chromatin occupancy during ovarian differentiation by chromatin immunoprecipitation (ChIP) followed by wholegenome sequencing ${ }^{42}$ at E14.5, a time where FOXL2 and RUNX1 expression fully overlaps (Fig. 3). We performed additional de novo motif analyses on the genomic regions bound by FOXL2 in the fetal ovary, and discovered that several other DNA motifs were co-enriched with FOXL2 DNA motif (Fig. 8a). RUNX DNA 


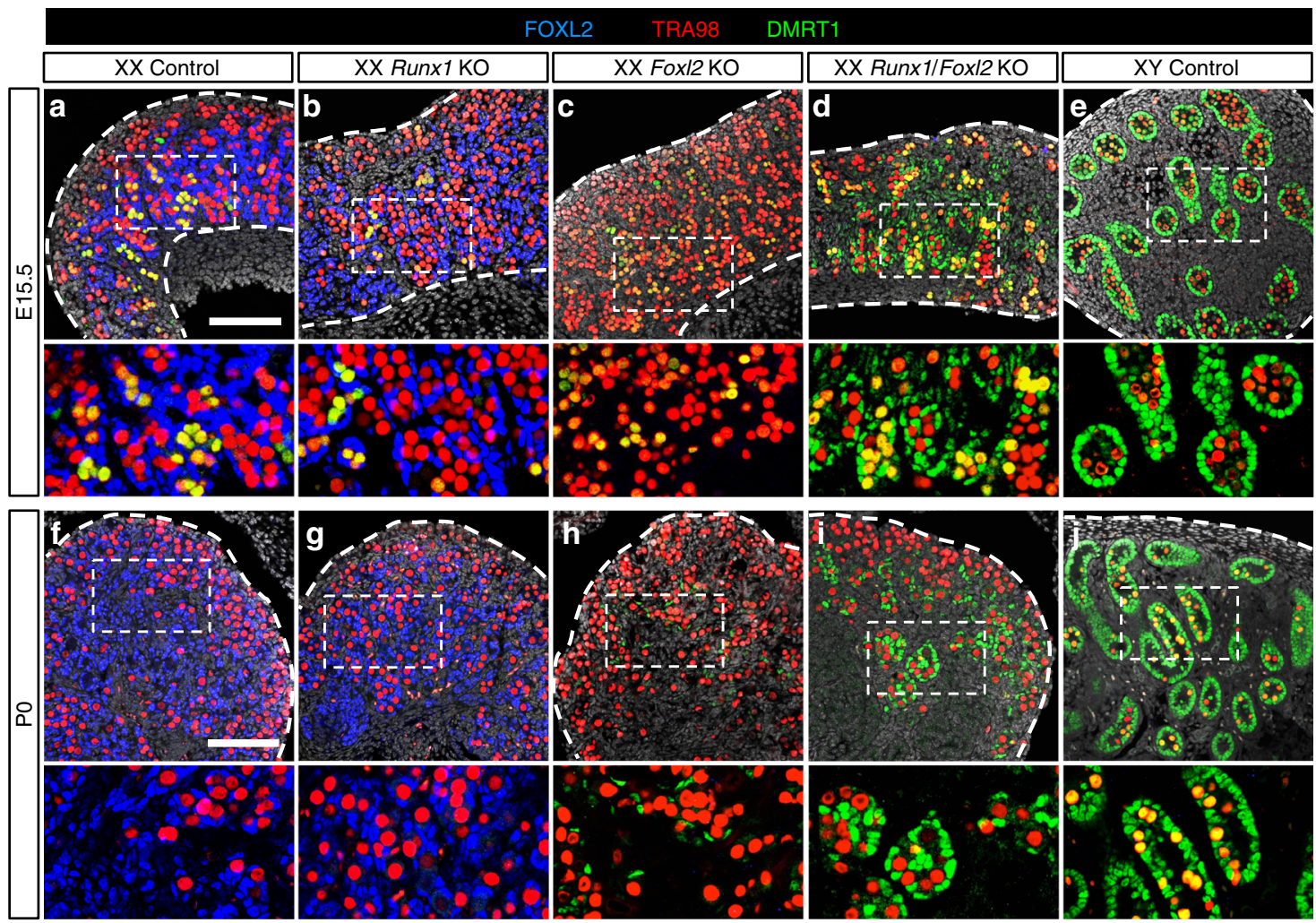

Fig. 5 Combined loss of Runx1 and FoxI2 masculinizes the fetal ovaries. a-j Immunofluorescence for Sertoli cell and germ-cell marker DMRT1, germ-cell marker TRA98, and granulosa cell marker FOXL2 in XX control, XX Runx1 KO, XX Fox/2 KO, XX Runx1/Fox/2 double knockout, and XY control gonads at E15.5 (a-e) and at birth (f-j). Controls correspond to wild-type littermates. The gray represents DAPI nuclear staining. Dotted lines outline the gonads. Higher magnifications are shown for the outlined boxes in $\mathbf{a}-\mathbf{e}$ and $\mathbf{f}-\mathbf{j}$, respectively. Scale bars: $100 \mu \mathrm{m}$. At least three independent biological replicates were analyzed and the images presented are representative of all replicates. Single-channel images are provided in Supplementary Figs. 3 and 4

motif was the second most significantly co-enriched motif. The other motifs were for CTCF, a factor involved in transcriptional regulation, enhancer insulation, and chromatin architecture ${ }^{43}$, and for the DNA motif recognized by members of the nuclear receptor family including liver receptor homolog-1 (LRH-1 encoded by Nr5a2) and SF1 (encoded by Nr5a1), a known cofactor of FOXL2 $2^{44,45}$. DNA motifs for TEAD transcription factors of the Hippo pathway, ETS, NFYA, and GATA4, another factor involved in gonad differentiation ${ }^{46}$, were also significantly enriched. The enrichment of RUNX motif with FOXL2-binding motif suggests that RUNX1, the only RUNX also expressed in pregranulosa cells, could bind similar genomic regions to FOXL2 in the fetal ovary. To confirm this hypothesis, we performed ChIPsequencing (ChIP-seq) for RUNX1 in E14.5 ovaries (Supplementary Data 6), the same stage as FOXL2 ChIP-seq ${ }^{42}$. The top de novo motif identified in RUNX1 ChIP-seq $(p<1 \mathrm{e}-559)$ matched the RUNX motif ${ }^{47}$ (Fig. 8b) and corresponded to the motif that was co-enriched with FOXL2 in FOXL2 ChIP-seq (Fig. 8a). A total of 10,494 RUNX1-binding peaks were identified in the fetal ovary, with the majority of the peaks located either in the gene body (Fig. $8 \mathrm{c} ; 25 \%$ exon and $22 \%$ intron) or close upstream of the transcription start site (TSS) (30\% Promoter: $<1$ $\mathrm{kb}$ of TSS; $12 \%$ Upstream: -10 to $-1 \mathrm{~kb}$ of TSS). Comparison of genome-wide chromatin binding of RUNX1 and FOXL2 in the fetal ovary revealed significant overlap: $54 \%(5619 / 10,494)$ of RUNX1 peaks overlapped with FOXL2 peaks (Fig. 8d).

The transcriptomic data from Runx1/Foxl2 DKO ovaries provided us a list of genes significantly changed as a result of the absence of Runx1, Foxl2, or both (Fig. 7). To identify potential direct target genes of RUNX1 or/and FOXL2, we focused on the
918 genes differentially expressed in Runx1/Foxl2 DKO ovaries and determined which genes were nearest to RUNX1 or/and FOXL2-binding peaks (Fig. 9a and Supplementary Data 7). More than $50 \%$ of these genes (492/918; Fig. 9a) were the closest gene to RUNX1 or/and FOXL2 peaks. Some of these genes were nearest to only FOXL2 peaks (116 genes in Fig. 9a). For example, Pla2r1, a transcript enriched in pre-granulosa cells ${ }^{40}$ and similarly downregulated in both Foxl2 KO and Runx1/Foxl2 DKO fetal ovaries (Fig. 9b), contained two FOXL2-specific peaks, one in the promoter and one in the first intron. On the other hand, 102 genes (Fig. 9a) had RUNX1-specific peaks near their genomic locations. For instance, Ryr2, another transcript enriched in pregranulosa cells ${ }^{40}$, was strongly downregulated in both Runx1 KO and Runx1/Foxl2 DKO fetal and newborn ovaries (Figs. $7 \mathrm{i}$ and 9c), and contained one RUNX1-specific peak in its intronic region. Finally, 274 genes were the closest genes to the peaks for both RUNX1 and FOXL2, with the majority of them (197 genes) nearest to overlapping peaks for RUNX1 and FOXL2 (Fig. 9a). Most of these genes were downregulated in Runx1/Foxl2 DKO ovaries (Supplementary Data 7). For instance, the pre-granulosa cell-enriched genes Fst and Itpr2, both downregulated in Runx1/ Foxl2 single and DKO ovaries (Figs. $7 \mathrm{~d}$ and $9 \mathrm{~d}$ ), contained common binding peaks for FOXL2 and RUNX1 (Fig. 9a, d). For Fst, this binding of RUNX1 and FOXL2 was located in its first intron, in the previously identified regulatory region that contributes to its expression ${ }^{42,48}$. On the other hand, Sertoli cell -enriched gene Dmrt1, which was strongly upregulated in Runx1/ Foxl2 DKO (Figs. 5 and 7i), contained a common binding site for FOXL2 and RUNX1 near its promoter (Fig. 9a). Taken together, our results reveal that RUNX1, a transcription factor expressed in 


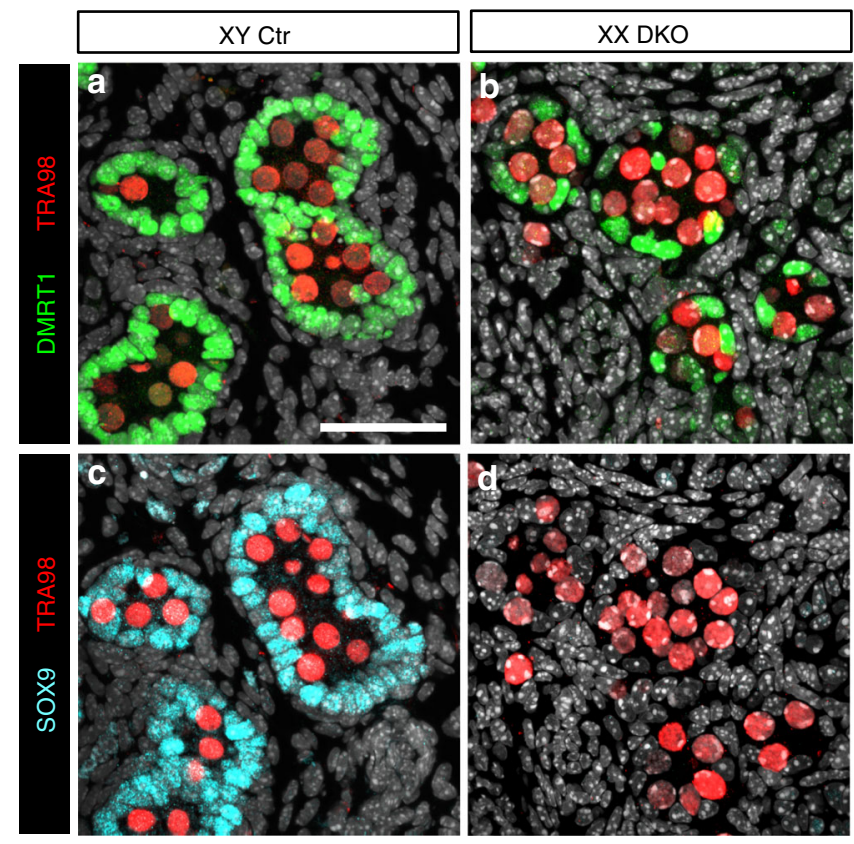

Fig. 6 SOX9 protein is not detected in Runx1/Fox/2 DKO newborn XX gonads. Immunofluorescence for Sertoli cell markers DMRT1 and SOX 9 and germ-cell marker TRA98 on consecutive sections in $X Y$ control (a, c) and XX Runx1/Fox/2 DKO gonads (b, d) at birth. Scale bar: $50 \mu \mathrm{m}$. At least three independent biological replicates were analyzed and the images presented are representative of all replicates

the fetal ovary of various vertebrate species, contributes to ovarian differentiation and maintenance of pre-granulosa cell identity through an interplay with FOXL2 that occurs at the chromatin level.

\section{Discussion}

The molecular events that specify pre-granulosa cell fate are complex and nonlinear, involving several signaling pathways that have redundant and complementary functions. This is in contrast to the fetal testis, where the molecular pathway driving its differentiation appears linear and sequential. Removal of one of the top pieces in the testis differentiation pathway has a domino effect that prevents induction of downstream events. This is exemplified by the complete gonadal sex reversal in gain- or lossof-function mouse models for SRY or SOX9, the two transcription factors responsible for the initiation of the testis morphogenesis $29,36,49$. This is not the case in the mouse ovary, where no single-gene loss/mutation results in a complete ovaryto-testis sex reversal. For instance, defects in the WNT4/RSPO1/ $\beta$-catenin pathway or loss of Foxl 2 causes a late or postnatal partial ovary-to-testis sex reversal, whereas the combined loss of Foxl2 and elements of the WNT4/RSPO1/ $\beta$-catenin pathway (Wnt4 or Rspo1) leads to ovary-to-testis sex reversal more pronounced than each single $\mathrm{KO}$ model in the mouse ${ }^{9,10}$. In this study, we demonstrated that Runx1 contributes to the molecular network controlling pre-granulosa cell differentiation. Loss of Runx1 in somatic cells of the ovaries altered ovarian transcriptome but did not affect ovarian morphogenesis during fetal development. In contrast, combined loss of Runx1 and Foxl2 compromised pre-granulosa cell identity. Loss of Runxl or Foxl2 affected common sets of genes, suggesting some redundancy in their functions in the fetal ovary. These transcriptomic changes were enhanced in the absence of both genes, reaching a threshold that masculinized the fetal ovary. Based on the phenotypic and transcriptomic analyses, we propose that FOXL2 is the dominant player and RUNX1 acts as a supporting player. For instance, global $\mathrm{KO}$ of Foxl2 in XX gonads results in postnatal sex reversal ${ }^{6}$, whereas the XX Runx1 single KO has no such impacts. In addition, loss of Foxl2 leads to transcriptomic changes more prominent than loss of Runxl in newborn ovaries, even though they affect common sets of genes. However, the additional loss of Runx 1 amplifies the transcriptomic changes caused by the loss of Foxl2, resulting in fetal masculinization. One of the most striking changes in Runx1/Foxl2 DKO ovaries is the expression of DMRT1 in the fetal supporting cells. DMRT1 is a key driver of Sertoli cell differentiation and testis development in various species $^{16,21}$. In the fly, doublesex $(d s x)$, an ortholog of mammalian DMRT1, controls testis differentiation ${ }^{16}$. Intriguingly, runt, the fly ortholog of RUNX1, tips the balance toward ovarian determination by antagonizing the testis-specific transcriptional regulation of $d s x^{22}$. In the mouse, testis differentiation is not controlled by DMRT1 but by SOX transcription factor SRY and its direct target SOX9. However, RUNX1 does not appear to contribute to the repression of the key pro-testis gene Sox 9 in the fetal ovary and SOX9 protein was not detected in Runx1/Foxl2 DKO ovary at birth. This is in contrast with the phenotype of Wnt4/Foxl2 DKO newborn ovaries where SOX9 was upregulated, and as a consequence the ovaries were more masculinized ${ }^{10}$. Overall, our findings suggest that slightly different pro-ovarian networks control the repression of the evolutionary conserved pro-testis genes Sox9 and Dmrt1: Sox9, which plays a primary role in Sertoli cell differentiation in the mouse, is repressed by an interplay between the WNT4/RSPO1/ $\beta$-catenin and FOXL2 ${ }^{9,10}$. On the other hand, Dmrt1, which has taken a secondary role in Sertoli cell differentiation in the mouse, is repressed by an interplay between RUNX1 and FOXL2. The fact that RUNX1 does not appear to control Sox 9 may be the reason why RUNX1 only plays a secondary role in granulosa cell differentiation/maintenance, in contrast to the dominant roles of the WNT pathway and FOXL2. It would be interesting to determine the role of RUNX1 in species for which DMRT1 plays a more prominent role in initiation of differentiation of the testis.

Seeking the mechanisms underlying the interplay between RUNX1 and FOXL2 in the regulation of pre-granulosa cell identity, we identified that RUNX DNA-binding motif is significantly co-enriched with FOXL2 motif in genomic regions bound by FOXL2 in the fetal ovary. The fact that RUNX1 genome-wide chromatin occupancy partially overlaps with FOXL2 in the fetal ovary, that RUNX1 and FOXL2 are expressed in the same cells at the time of the ChIP-seqs, that their loss affects common set of genes, and that the DKO results in gonad masculinization, altogether support the model in which RUNX1 and FOXL2 jointly occupy common chromatin regions that control the maintenance of pre-granulosa cell identity. By themselves, RUNX proteins are weak transcription factors and they require other transcriptional regulators to function as either repressors or activators of transcription ${ }^{26}$. Interplay between RUNX1 and several members of the forkhead transcription factor family has been documented in different tissues. For instance, RUNX1 is a co-activator of FOXO3 in hepatic cells ${ }^{50}$. Similarly, an interplay between RUNX1 and FOXO1/FOXO3 was demonstrated in breast epithelial cells where a subset of FOXO target genes were jointly regulated with RUNX1 ${ }^{51}$. Another forkhead protein, FOXP3, acts with RUNX1 to control gene expression in $\mathrm{T}$ cells ${ }^{52}$ and breast epithelial cells $\mathrm{s}^{53}$. Such cooperation in various tissues suggest that the interplay between RUNXs and forkhead transcription factors maybe an evolutionary conserved phenomenon. It would be interesting to determine whether the forkhead protein FOXL2 is also able to physically interact with RUNX1 in the fetal ovaries and how this interaction occurs. Our data and single-cell sequencing data ${ }^{27}$ demonstrate that RUNX1 starts to 
a

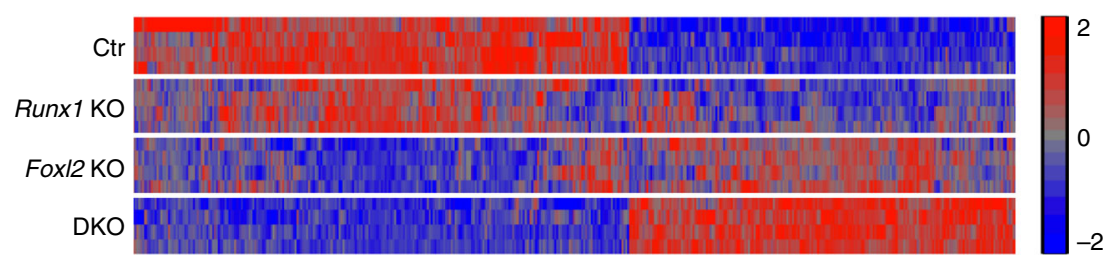

b

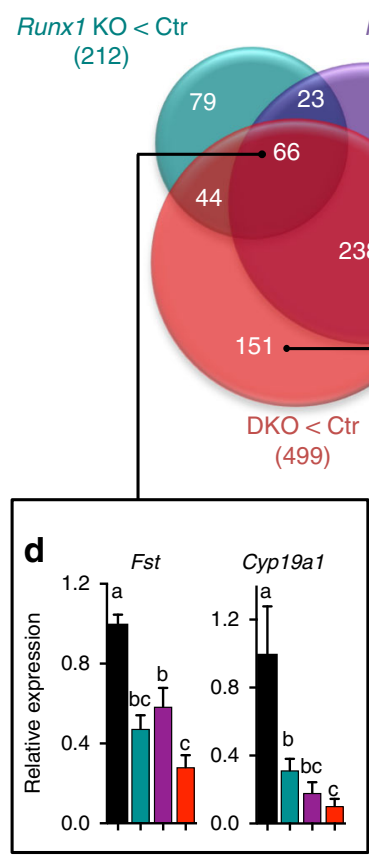

Foxl2 $\mathrm{KO}<\mathrm{Ctr}$ (459)

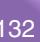

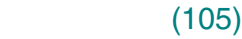

$\operatorname{Runx1}$
$(105)$

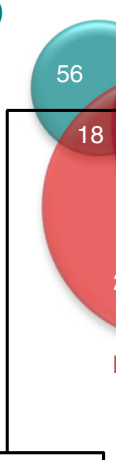

Genes upregulated
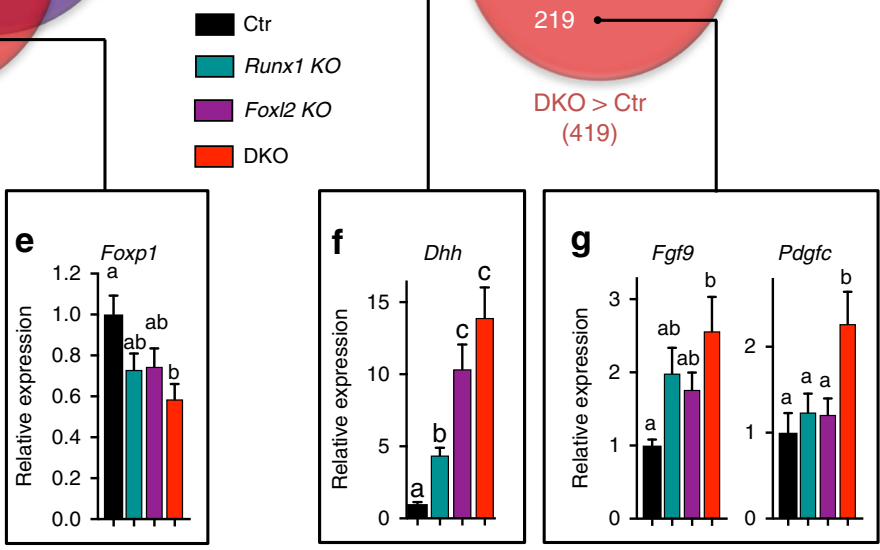

h
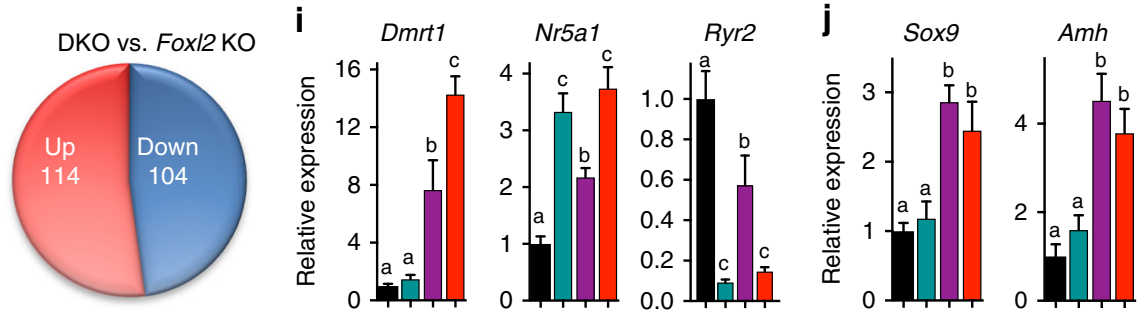

Fig. 7 Transcriptomic analysis of XX Runx1/Fox/2 single and double KO ovaries. a Heat map for the 918 genes differentially expressed in XX Runx $1 /$ Fox $/ 2$ double knockout (DKO) vs. XX control (Ctr) gonads at birth. The heat map shows the expression of these 918 genes in XX control, Runx $1 \mathrm{KO}$, Foxl2 KO, and Runx1/Fox/2 DKO gonads (microarray; $n=4$ /genotype; one-way ANOVA; fold change $>1.5, p<0.05$ ). Controls correspond to XX wild-type littermates. b, c Venn diagram comparing the genes downregulated (b) or upregulated (c) in XX Runx1 KO (green circle), XX Fox/2 KO (purple circle), and XX Runx1/ Fox 12 DKO (red circle) gonads at birth. $\mathbf{d}-\mathbf{g}$ Validation by quantitative PCR of genes identified in the Venn diagrams as significantly downregulated in all three KO (d), or only in Runx1/Fox/2 DKO (e), or significantly upregulated in all in all three KO (f) or only in Runx1/Fox/2 DKO (g). h, i Identification of the genes differentially expressed in XX Runx1/Fox/2 DKO vs. XX Fox/2 KO gonads and validation of candidate genes by quantitative PCR. $\mathbf{j}$ Expression of Sox9 and Amh is not changed in XX Runx1/Fox/2 DKO compared with XX Fox/2 KO gonads. For all the qPCR data, values are presented as mean \pm SEM $(n=5 /$ genotype). One-way ANOVA, $p<0.05$. Bars with different letters $(\mathbf{a}, \mathbf{b}, \mathbf{c})$ are significantly different. Source data are provided as a Source Data file

be expressed in the supporting cells earlier than FOXL2. In other tissues, it was demonstrated that RUNX1 can function as a pioneer factor that allows chromatin remodeling and recruitment of other factors to control gene expression and cell fate $\mathrm{e}^{54}$. Therefore, it is possible that RUNX1 is first recruited to chromatin regions and the presence of RUNX1 facilitates the recruitment of FOXL2 to maintain pre-granulosa cell identity. Finally, in addition to the genes co-regulated by FOXL2 and RUNX1, we identified genes that were specifically mis-expressed in the absence of Runx1 but not Foxl2. Genome-wide analyses of RUNX1 binding in the fetal ovary also identified genomic regions bound by RUNX1 but not
FOXL2. These results suggest that RUNX1 could also contribute to ovarian development or function independently of FOXL2.

RUNX1 contributes to cell-fate determination in various developmental processes such as hematopoiesis and hair follicle development. Depending on its interplay with other signal transduction pathways or co-factors, RUNX1 controls which path the precursor cells take when they are at the crossroad between cell proliferation/renewal and lineage-specific commitment ${ }^{25}$. We discovered that Runx1 has an ovary-biased expression during gonad differentiation in various vertebrate species, including turtle, rainbow trout, goat, mouse, and human. In mouse 
FOXL2 ChIP-seq

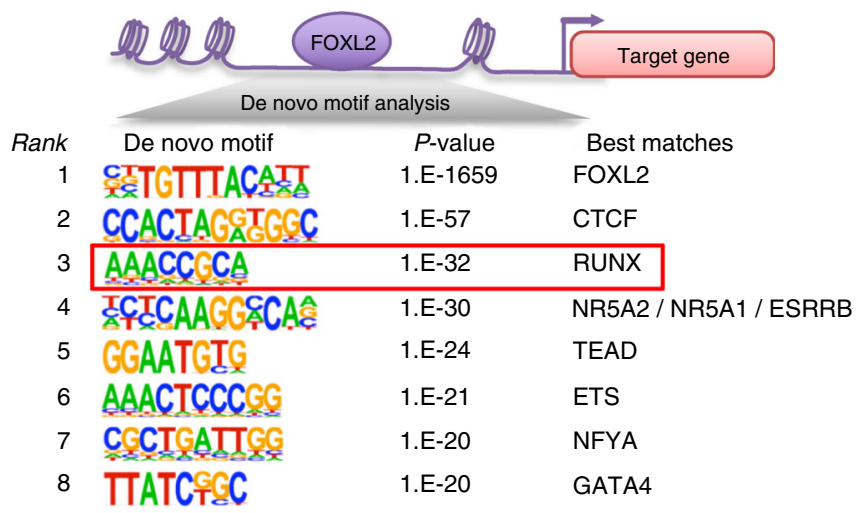

b

RUNX1 ChIP-seq

C

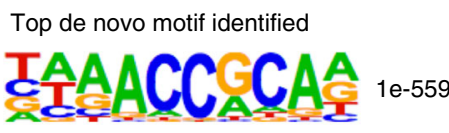

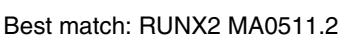
SAAACCACA

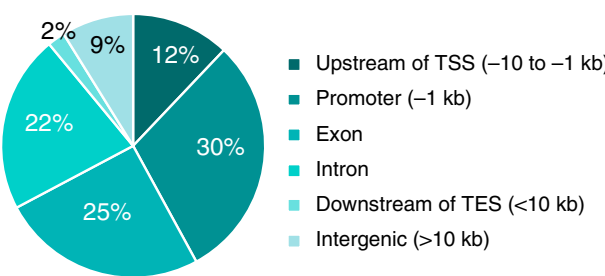

d

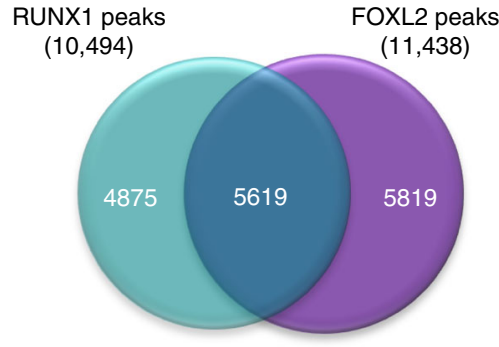

Fig. 8 RUNX1 and FOXL2 exhibit overlaps in chromatin binding in fetal ovaries. a de novo motif analysis of FOXL2 peaks identifies enrichment of RUNX motif along with FOXL2 motif in E14.5 ovaries. b The top de novo motif for RUNX1 ChIP-seq in E14.5 ovaries corresponds to a RUNX motif. c Distribution of genomic location of the 10,494 RUNX1-binding peaks. TSS, transcription start site; TES, transcription end site. d Comparison of RUNX1 (10,494 peaks) and FOXL2 (11,438 peaks) chromatin occupancy in E14.5 ovaries

embryonic gonads, Runx 1 is first detected in the supporting cells in a non-sexually dimorphic way at the onset of sex determination. Although its expression is maintained in the ovary, Runx1 appears to be actively repressed in the testis between E11.5 and E12.5 as the supporting cells commit to Sertoli cell fate. The suppression of Runxl in the fetal testis is corroborated by previously published data from a time-course transcriptomic analysis during early gonad development ${ }^{55}$ and single-cell sequencing analysis of SF1+ progenitor cells ${ }^{27,56}$. The time course of Sertoli cell differentiation at the single-cell level revealed that Runx1 follows a similar spatiotemporal pattern of expression with $S r y^{56}$. In the mouse, Sry expression in Sertoli cells is quickly turned off after the initiation of testis differentiation and it is suspected that the repression of Sry is due to a negative feedback loop by downstream pro-testis genes. The similar pattern of downregulation of Runx1 in the testis after E11.5 raises the possibility that Runx1 is downregulated by a similar signaling pathway. Regulation of Runxl gene expression is complex and several enhancers that confer tissue-specific expression have been identified $^{57}$. It remains to be determined how Runxl expression is controlled in the gonads and how it is actively repressed in the fetal testis.
In contrary to the testes, fetal ovaries maintain expression of Runxl in the supporting cells as they differentiate into pregranulosa cells. During ovarian differentiation, granulosa cells arise from two different waves: the first cohort of granulosa cells arises from the bipotential supporting cell precursors that differentiate into either Sertoli cells or pre-granulosa cells during sex determination $^{58}$. The second wave of granulosa cells that eventually populate the cortical region of the ovary appears later in gestation. This second wave arises from LGR5+ cells of the ovarian surface epithelium that ingress into the ovary from E15.5 to postnatal day 4 and eventually become LGR5-/FOXL2+ granulosa cells ${ }^{30,31}$. This timing of establishment of the second cohort of granulosa cells correlates with the expression of Runx1EGFP in a subset of cells in the surface epithelium and in granulosa cells of the ovarian cortex at E16.5 and birth. These results suggest that Runx1 also marks granulosa cell precursors that will give rise to the second wave of FOXL2+ granulosa cells in the cortex. Therefore, both expression at onset of sex determination and at the surface epithelium/cortex at the time of the second wave of granulosa cells recruitment suggest that Runx1 is activated in cells that are primed to become supporting/ granulosa cells. 
a
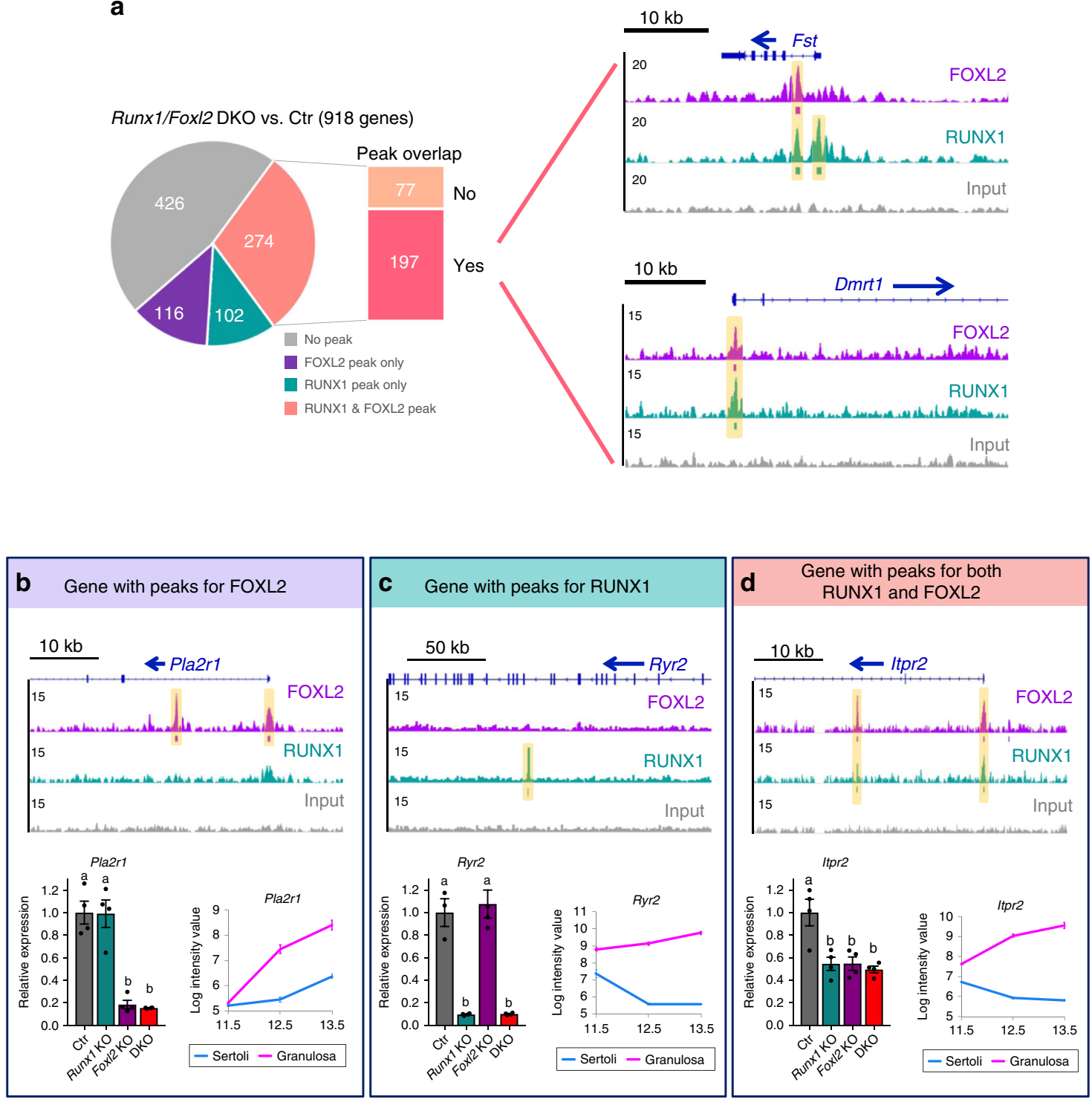

Fig. 9 Identification of potential direct target genes for RUNX1 and/or FOXL2. a Pie-chart identifying the genes significantly changed in XX Runx1/Fox/2 DKO gonads that are nearest to peaks for FOXL2 and/or RUNX1. Genome browser view of two key genes significantly changed in XX Runx1/Fox/2 DKO gonads and bound by RUNX1 and FOXL2 in E14.5 ovaries. Blue arrows: gene orientation; orange highlighted area: significant binding peaks identified by HOMER. b-d Examples of genes affected in XX Runx1/Fox/2 DKO gonads and bound by FOXL2 or/and RUNX1. For each gene, we show the genome browser view of RUNX1 and/or FOXL2 binding in E14.5 ovaries, the gene expression by quantitative PCR in XX Runx1/Fox/2 single and double knockouts gonads at E15.5 ( $n=4$ /genotype; mean \pm SEM; one-way ANOVA, $p<0.05$; bars with different letters (a, b) are significantly different) and the gene expression in fetal Sertoli and granulosa cells from E11.5 to E13.540. Source data are provided as a Source Data file

Multiple transcription factors often form complex genetic regulatory networks that control cell-fate determination. Genomic sequence motifs or cis-regulatory elements for the supporting cell lineage in the testis were identified by combined analyses of SOX9 and DMRT1 ChIP-seq, and by motif prediction ${ }^{59}$. These "Sertoli cell signatures" are composed of binding motifs for transcription factors critical for Sertoli cell differentiation, including SOX9, GATA4, and DMRT1. These Sertoli cell signatures, present in mammals and other vertebrates, could represent a conserved regulatory code that governs the cascade of Sertoli cell differentiation, regardless of whether it primarily relies on SOX transcription factors such as SRY in mammals or on DMRT1 such as in several vertebrate species. Similarly, one would expect the presence of conserved "granulosa cell signature" genomic regions that confers granulosa cell differentiation. As FOXL2 is a highly conserved gene in granulosa cell differentiation in vertebrates, we used FOXL2 as an anchor factor to identify other factors that could take part in the regulatory network controlling granulosa cell differentiation/function. Unbiased analyses of the motifs co-enriched with FOXL2 motif in the fetal ovary identified the RUNX motif as one of the most coenriched motifs. In addition to the RUNX motif, motifs for CTCF, nuclear receptors SF1/LRH-1/ESRRB, and transcription factors TEADs and GATAs were also significantly enriched with FOXL2 consensus motif in FOXL2-bound chromatin regions. For many of these transcription factors, their potential role in gonad differentiation is unknown or limited. For example, the transcription factors of the TEAD family belong to the Hippo pathway, which is involved in the regulation of Sertoli cell gene expression in the fetal gonads ${ }^{60}$. However, the potential involvement of the hippo pathway in granulosa cell differentiation has not been investigated. 
In conclusion, we identified RUNX1 as a transcription factor involved in pre-granulosa cell differentiation/maintenance. RUNX1 first delineates the supporting cell lineage and then becomes pre-granulosa cell-specific during gonad development. RUNX1 plays redundant roles with FOXL2 through binding of common chromatin regions and control of common sets of genes to maintain pre-granulosa cell identity in the fetal ovary. Our findings provide insights into the genomic control of granulosa cell differentiation and pave the way for the identification of transcription factors and cis-signatures contributing to the fate determination of granulosa cells and the consequent formation of a functional ovary.

\section{Methods}

Mouse models. $\operatorname{Tg}($ Runx 1 -EGFP) reporter mouse was purchased from MMRRC (MMRRC_010771-UCD) and CD-1 mice were purchased from Charles River (stock number 022). Runx $1^{+/-}$(B6.129S-Runx $\left.1^{\text {tm } 1 S p e} / \mathrm{J}\right)$ and Runxilf $(\mathrm{B} 6.129 \mathrm{P} 2$ Runx $1^{\text {tm } 1 \text { Tani }} / \mathrm{J}$ ) mice were purchased from the Jackson Laboratory (stock numbers 005669 and 008772 , respectively). Sf1-Cre ${ }^{\mathrm{Tg} / \mathrm{Tg}} \mathrm{mice}^{34}$ (B6D2-Tg(Nr5a1-cre)2Klp) were provided by late Dr. Keith Parker and Foxl2 $2^{+1-}$ mice $^{61}($ B6;129-Foxl2 < tm 1 Gpil $>$ ) were provided by Dr. David Schlessinger (National Institute on Aging), respectively. Runx1 KO mice $\left(S f 1 C r e^{+/ T g}\right.$; Runx $\left.1^{f /-}\right)$ were generated by crossing Runx $1^{f l f}$ females with Sf1-Cre $e^{+/ T g}$; Runx $1^{+/-}$males. Controls were Sf1-Cre ${ }^{+/}$; Run $x 1^{+/ f}$ littermates. Runx1/Foxl2 DKO mice (Sf1Cre ${ }^{+/ T g}$; Run $x 1^{f /-}$; Foxl2 ${ }^{-/-}$)

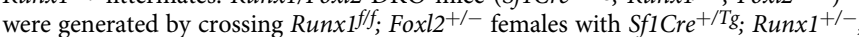
Foxl2 ${ }^{+/-}$males. This cross also generated the single KOs for Runxl $\left(\mathrm{SflCre} \mathrm{C}^{+/ \mathrm{Tg}}\right.$; Runx $^{f /-}$; Foxl2 $\left.{ }^{+/+}\right)$and Foxl2 (SflCre ${ }^{+/+}$; Runx $1^{f /+} ;$ Foxl2 $\left.{ }^{-/-}\right)$, and control littermates (Sf1-Cre ${ }^{+/+}$; Runx1 $1^{+/ f}$; Foxl2 ${ }^{+/+}$). Time-mating was set up by housing female mice with male mice overnight and the females were checked for the presence of vaginal plug the next morning. The day when the vaginal plug was detected was considered embryonic day or E0.5. All experiments were performed on at least four animals for each genotype. All animal procedures were approved by the National Institutes of Health Animals Care and Use Committee, and were performed in accordance with an approved National Institute of Environmental Health Sciences animal study proposal.

Immunofluorescences. For the $\operatorname{Tg}($ Runxl-EGFP) mice, gonads were collected and fixed in $4 \%$ paraformaldehyde for $1-2 \mathrm{~h}$ at room temperature. Immunofluorescence experiments were performed on whole gonads at E11.5 and E12.5, and on sections for E14.5, E15.5, E16.5, and P0 (birth) gonads. The EGFP was detected in wholemount gonads by direct fluorescent imaging and an anti-GFP antibody was used for immunofluorescences on sections. For the different $\mathrm{KO}$ models, gonads were fixed in $4 \%$ paraformaldehyde overnight at $4{ }^{\circ} \mathrm{C}$ and immunofluorescence experiments were performed on paraffin sections of E14.5, E15.5, and P0 gonads as previously described ${ }^{62}$. Briefly, the slides were rehydrated and citrate-based antigen retrieval was performed. The samples (slides or whole-mount samples) were blocked in blocking buffer ( $5 \%$ donkey serum/0.1\% Triton X-100 in phosphatebuffered saline (PBS)) for $1 \mathrm{~h}$ at room temperature. The samples were then incubated overnight at $4{ }^{\circ} \mathrm{C}$ in the primary antibodies diluted in blocking buffer. The next day, the samples were washed three times in $0.1 \%$ Triton X-100 in PBS and were incubated for $1 \mathrm{~h}$ at room temperature in the secondary antibodies diluted in blocking buffer. The samples were then washed and counterstained with DAPI (4',6-diamidino-2-phenylindole). The antibodies used in this study are listed in Supplementary Table 1 . Whole gonads and sections were imaged under a Leica DMI4000 confocal microscope. For all immunofluorescence experiments, at least three independent biological replicates were analyzed and the image presented in the figures were representative of all replicates.

Real-time PCR analysis in the mouse. For the time-course kinetics of Runx expression, fetal gonads from CD-1 embryos at embryonic day E11.5, E12.5, E13.5, E14.5, E16.5, E18.5, and postnatal day P3 were separated from the mesonephros and snap-frozen. For each stage, three biological replicates were collected, with six gonads/replicate for the E11.5 stage and three gonads/replicate for the other stages. For Runx1 KO analysis, control and $\mathrm{KO}$ ovaries were collected at E14.5 ( $n=4$ biological replicates/genotype). For Runx1/Foxl2 DKO analysis, control, Runx1, and Foxl2 single and DKO ovaries were collected at E15.5 $(n=4 /$ genotype $)$ and P0 ( $n=5 /$ genotype). For all experiments, total RNA was isolated for each replicate using PicoPure RNA isolation kit (Arcturus, Mountain View, CA). RNA quality and concentration were determined using the Nanodrop 2000c and 300-400 ng of RNA was used for cDNA synthesis with the Superscript II cDNA synthesis system (Invitrogen Corp., Carlsbad, CA). Gene expression was analyzed by real-time PCR using Bio-Rad CFX96TM Real-Time PCR Detection system. Gene expression was normalized to Gapdh. The Taqman probes and primers used to detect transcript expression are listed in Supplementary Tables 2 and 3. Data were analyzed using Prism GraphPad Software by unpaired Student's $t$-test or by ANOVA $p<0.05$. Values are presented as mean \pm SEM.
Runx1 expression in other species. For the rainbow trout, Runx1 expression during gonadal development was assessed by quantitative $\mathrm{PCR}^{63}$. Species-specific primers used are listed in Supplementary Table 3. For the red-eared slider turtle, Runxl expression during gonadal development was assessed at Female-Promoting Temperature of $31^{\circ} \mathrm{C}$ and at Male-Promoting Temperature of $26^{\circ} \mathrm{C}$ by RNAsequencing (RNA-seq) ${ }^{64}$. For the goat, Runxl expression during gonadal development was assessed by quantitative PCR and two to three biological replicates were used for each stage of development ${ }^{65}$. Values are presented as mean \pm SD. All goat-handling procedures were conducted in compliance with the guidelines on the Care and Use of Agricultural Animals in Agricultural Research and Teaching in France (Authorization number 91-649 for the Principal Investigator, and national authorizations for all investigators. Approval from the Ethics Committee: 12/045) For the human, Runxl expression during gonadal development was assessed by RNA-seq. Human fetuses (6-12 GW) were obtained from legally induced normally progressing terminations of pregnancy performed in Rennes University Hospital in France. Tissues were collected with women's written consent, in accordance with the legal procedure agreed by the National agency for biomedical research (authorization \#PFS09-011; Agence de la Biomédecine) and the approval of the Local ethics committee of Rennes Hospital in France (advice \# 11-48).

Microarray analysis. Gene expression analysis of control, Runx1 KO, Foxl2 KO, and Runx1/Foxl2 DKO ovaries was conducted using Affymetrix Mouse Genome 4302.0 GeneChip ${ }^{\circledR}$ arrays (Affymetrix, Santa Clara, CA) on four biological replicates (one P0 gonad per replicate) for each genotype. Fifty nanograms of total RNA were amplified and labeled as directed in the WT-Ovation Pico RNA Amplification System and Encore Biotin Module protocols. Amplified biotin-aRNA ( $4.6 \mu \mathrm{g})$ was fragmented and hybridized to each array for $18 \mathrm{~h}$ at $45^{\circ} \mathrm{C}$ in a rotating hybridization. Array slides were stained with streptavidin/phycoerythrin utilizing a double-antibody staining procedure and then washed for antibody amplification according to the GeneChip Hybridization, Wash and Stain Kit, and user manual following protocol FS450-0004. Arrays were scanned in an Affymetrix Scanner 3000 and data were obtained using the GeneChip ${ }^{\circledR}$ Command Console Software (AGCC; Version 3.2) and Expression Console (Version 1.2). Microarray data have been deposited in GEO under accession code GSE129038. Gene expression analyses were conducted with Partek software (St. Louis, Missouri) using a one-way ANOVA comparing the Robust Multichip Average (RMA)-normalized log2 intensities. A full dataset Excel file containing the normalized $\log 2$ intensity of all genes for each genotype and a graphic view of their expression is provided in Supplementary Data 2. In order to identify differentially expressed genes, ANOVA was used to determine whether there was a statistical difference between the means of groups and the gene lists were filtered with $p<0.05$ and fold-change cutoff of 1.5 . The heat map was created comparing the genes that were significantly different between control and Runx1/Foxl2 DKO ovaries. Venn diagrams were generated in Partek by comparing gene symbols between the lists of genes differentially expressed.

ChIP-seq assays and analysis. Ovaries from E14.5 CD-1 embryos were separated from the mesonephros, snap-frozen, and stored at $-80^{\circ} \mathrm{C}$. RUNX1 ChIP-seq experiments and analyses in E14.5 ovaries were performed using the same protocol than FOXL2 ChIP-seq ${ }^{42}$. Two independent ChIP-seq experiments were performed by Active Motif, Inc., using 20-30 $\mu \mathrm{g}$ of sheared chromatin from pooled embryonic ovaries $\left(n=100-120\right.$ ovaries/ChIP) and $10 \mu \mathrm{l}$ of RUNX1 antibody ${ }^{66}$ (provided by Drs Yoram Groner and Ditsa Levanon, the Weizmann Institute of Science, Israel) ChIP-seq libraries were sequenced as single-end 75-mers by Illumina NextSeq 500 , then filtered to retain only reads with average base quality score $>20$. Reads were mapped against the mouse mm10 reference genome using Bowtie v1.2 with parameter "-m 1" to collect only uniquely mapped hits. Duplicate mapped reads were removed using Picard tools MarkDuplicates.jar (v1.110). The number of uniquely mapped non-duplicate reads for each biological replicate was $8,932,674$ and 15,036,698. After merging the replicate datasets, binding regions were identified by peak calling using HOMER v. $4.9^{67}$ with a false discovery rate $<1 \mathrm{e}-5$. Called peaks were subsequently re-defined as 300 mers centered on the called peak midpoints and filtered for a fourfold enrichment over input and over local signal. Genomic distribution of RUNX1-bound regions was determined based on Refseq gene models as downloaded from the UCSC Genome Browser as of 9 August 2017. Enriched motifs were identified using HOMER findMotifsGenome.pl de novo motif analysis with parameter "-size given". For RUNX1 and FOXL2 ChIP-seq comparisons, binding peaks that had at least $1 \mathrm{bp}$ in common were considered overlapping. Peaks were assigned to the nearest gene based on RefSeq. Gene lists were analyzed for enrichment using the online tool EnrichR ${ }^{68}$. The ChIP-seq data are available in the ReproGenomics Viewer (https://rgv.genouest.org) ${ }^{69,70}$ and Gene Expression Omnibus (GSE128767; http://www.ncbi.nlm.nih.gov/geo/).

Reporting summary. Further information on research design is available in the Nature Research Reporting Summary linked to this article.

\section{Data availability}

The authors declare that all data supporting the findings of this study are available within the article and its Supplementary Information files or from the corresponding author 
upon reasonable request. Microarray and ChIP-seq data generated in this study have been deposited in the GEO database under accession codes GSE129038 and GSE128767, respectively. The ChIP-seq data are available in the ReproGenomics Viewer (https://rgv. genouest.org). Raw data underlying all reported mean values in graphs are provided in the Source Data File. All other relevant data supporting the key findings of this study are available in the Supplementary Information files. The source data underlying Figs. 1, $4 \mathrm{~d}-\mathrm{e}, 4 \mathrm{~h}, 7 \mathrm{~d}-\mathrm{g}, 7 \mathrm{i}-\mathrm{j}$, and $9 \mathrm{~b}-\mathrm{d}$ are provided as a Source Data file.

Received: 24 April 2019; Accepted: 4 September 2019;

Published online: 11 November 2019

\section{References}

1. Capel, B. Vertebrate sex determination: evolutionary plasticity of a fundamental switch. Genetics 18, 675-689 (2017).

2. Chassot, A. A. et al. Activation of beta-catenin signaling by Rspol controls differentiation of the mammalian ovary. Hum. Mol. Genet 17, 1264-1277 (2008).

3. Tomizuka, K. et al. R-spondin1 plays an essential role in ovarian development through positively regulating Wnt-4 signaling. Hum. Mol. Genet. 17, 1278-1291 (2008).

4. Vainio, S., Heikkila, M., Kispert, A., Chin, N. \& McMahon, A. P. Female development in mammals is regulated by Wnt-4 signalling. Nature 397, 405-409 (1999).

5. Harris, A. et al. ZNRF3 functions in mammalian sex determination by inhibiting canonical WNT signaling. Proc. Natl Acad. Sci. USA 115 5474-5479 (2018).

6. Ottolenghi, C. et al. Foxl2 is required for commitment to ovary differentiation. Hum. Mol. Genet. 14, 2053-2062 (2005).

7. Schmidt, D. et al. The murine winged-helix transcription factor Foxl2 is required for granulosa cell differentiation and ovary maintenance. Development 131, 933-942 (2004).

8. Uhlenhaut, N. H. et al. Somatic sex reprogramming of adult ovaries to testes by FOXL2 ablation. Cell 139, 1130-1142 (2009).

9. Auguste, A. et al. Loss of R-spondin1 and Foxl2 amplifies female-to-male sex reversal in XX mice. Sex. Dev. 5, 304-317 (2011)

10. Ottolenghi, C. et al. Loss of Wnt4 and Foxl2 leads to female-to-male sex reversal extending to germ cells. Hum. Mol. Genet. 16, 2795-2804 (2007)

11. Herpin, A. \& Schartl, M. Plasticity of gene-regulatory networks controlling sex determination: of masters, slaves, usual suspects, newcomers, and usurpators. EMBO Rep. 16, 1260-1274 (2015).

12. Crisponi, L. et al. The putative forkhead transcription factor FOXL2 is mutated in blepharophimosis/ptosis/epicanthus inversus syndrome. Nat. Genet. 27, 159-166 (2001).

13. Boulanger, L. et al. FOXL2 is a female sex-determining gene in the goat. Curr. Biol. 24, 404-408 (2014).

14. Li, M. et al. Efficient and heritable gene targeting in tilapia by CRISPR/Cas9. Genetics 197, 591-599 (2014).

15. Bertho, S. et al. The unusual rainbow trout sex determination gene hijacked the canonical vertebrate gonadal differentiation pathway. Proc. Natl Acad. Sci. USA 115, 12781-12786 (2018)

16. Raymond, C. S. et al. Evidence for evolutionary conservation of sexdetermining genes. Nature 391, 691-695 (1998).

17. Matsuda, M. et al. DMY is a Y-specific DM-domain gene required for male development in the medaka fish. Nature 417, 559-563 (2002).

18. Nanda, I. et al. A duplicated copy of DMRT1 in the sex-determining region of the Y chromosome of the medaka, Oryzias latipes. Proc. Natl Acad. Sci. USA 99, 11778-11783 (2002).

19. Matson, C. K. et al. DMRT1 prevents female reprogramming in the postnatal mammalian testis. Nature 476, 101-104 (2011).

20. Mello, M. P. et al. Novel DMRT1 3'UTR+11insT mutation associated to XY partial gonadal dysgenesis. Arq. Bras. Endocrinol. Metab. 54, 749-753 (2010).

21. Raymond, C. S., Murphy, M. W., O’Sullivan, M. G., Bardwell, V. J. \& Zarkower, D. Dmrt1, a gene related to worm and fly sexual regulators, is required for mammalian testis differentiation. Genes Dev. 14, 2587-2595 (2000).

22. Duffy, J. B. \& Gergen, J. P. The Drosophila segmentation gene runt acts as a position-specific numerator element necessary for the uniform expression of the sex-determining gene sex-lethal. Genes Dev. 5, 2176-2187 (1991).

23. Kramer, S. G., Jinks, T. M., Schedl, P. \& Gergen, J. P. Direct activation of sexlethal transcription by the Drosophila Runt protein. Development 126, 191-200 (1999).

24. Nef, S. et al. Gene expression during sex determination reveals a robust female genetic program at the onset of ovarian development. Dev. Biol. 287, 361-377 (2005).

25. Groner Y., et al. RUNX Proteins in Development and Cancer (Springer, 2017).
26. Chuang, L. S., Ito, K. \& Ito, Y. RUNX family: regulation and diversification of roles through interacting proteins. Int J. Cancer 132, 1260-1271 (2013).

27. Stevant, I. et al. Dissecting cell lineage specification and sex fate determination in gonadal somatic cells using single-cell transcriptomics. Cell Rep. 26, 3272-3283.e3273 (2019)

28. Gong, S. et al. A gene expression atlas of the central nervous system based on bacterial artificial chromosomes. Nature 425, 917-925 (2003).

29. Koopman, P., Gubbay, J., Vivian, N., Goodfellow, P. \& Lovell-Badge, R. Male development of chromosomally female mice transgenic for Sry. Nature 351, 117-121 (1991)

30. Mork L., et al. Temporal differences in granulosa cell specification in the ovary reflect distinct follicle fates in mice. Biol. Reprod. 86, 37 (2012).

31. Rastetter, R. H. et al. Marker genes identify three somatic cell types in the fetal mouse ovary. Dev. Biol. 394, 242-252 (2014).

32. Okuda, T., vanDeursen, J., Hiebert, S. W., Grosveld, G. \& Downing, J. R. AML1, the target of multiple chromosomal translocations in human leukemia, is essential for normal fetal liver hematopoiesis. Cell 84, 321-330 (1996).

33. Wang, Q. et al. Disruption of the Cbfa2 gene causes necrosis and hemorrhaging in the central nervous system and blocks definitive hematopoiesis. Proc. Natl Acad. Sci. USA 93, 3444-3449 (1996).

34. Bingham, N. C., Verma-Kurvari, S., Parada, L. F. \& Parker, K. L. Development of a steroidogenic factor 1/Cre transgenic mouse line. Genesis 44, 419-424 (2006).

35. Lei, N. et al. Sex-specific differences in mouse DMRT1 expression are both cell type- and stage-dependent during gonad development. Biol. Reprod. 77, 466-475 (2007).

36. Chaboissier, M. C. et al. Functional analysis of Sox 8 and Sox 9 during sex determination in the mouse. Development 131, 1891-1901 (2004).

37. Vidal, V. P., Chaboissier, M. C., de Rooij, D. G. \& Schedl, A. Sox 9 induces testis development in XX transgenic mice. Nat. Genet. 28, 216-217 (2001).

38. Jorgez, C. J., Klysik, M., Jamin, S. P., Behringer, R. R. \& Matzuk, M. M. Granulosa cell-specific inactivation of follistatin causes female fertility defects. Mol. Endocrinol. 18, 953-967 (2004).

39. Britt, K. L. et al. The ovarian phenotype of the aromatase knockout (ArKO) mouse. J. Steroid Biochem. Mol. Biol. 79, 181-185 (2001).

40. Jameson, S. A. et al. Temporal transcriptional profiling of somatic and germ cells reveals biased lineage priming of sexual fate in the fetal mouse gonad. PLoS Genet. 8, e1002575 (2012).

41. Colvin, J. S., Green, R. P., Schmahl, J., Capel, B. \& Ornitz, D. M. Male-tofemale sex reversal in mice lacking fibroblast growth factor 9. Cell 104, 875-889 (2001).

42. Nicol, B. et al. Genome-wide identification of FOXL2 binding and characterization of FOXL2 feminizing action in the fetal gonads. Hum. Mol. Genet. 27, 4273-4287 (2018).

43. Ong, C. T. \& Corces, V. G. CTCF: an architectural protein bridging genome topology and function. Nat. Rev. Genet. 15, 234-246 (2014).

44. Park, M. et al. FOXL2 interacts with steroidogenic factor-1 (SF-1) and represses SF-1-induced CYP17 transcription in granulosa cells. Mol. Endocrinol. 24, 1024-1036 (2010).

45. Yang, W. H., Gutierrez, N. M., Wang, L. Z., Ellsworth, B. S. \& Wang, C. M. Synergistic activation of the Mc2r promoter by FOXL2 and NR5A1 in mice. Biol. Reprod. 83, 842-851 (2010).

46. Manuylov, N. L., Smagulova, F. O., Leach, L. \& Tevosian, S. G. Ovarian development in mice requires the GATA4-FOG2 transcription complex. Development 135, 3731-3743 (2008)

47. Meyers, S., Downing, J. R. \& Hiebert, S. W. Identification of AML-1 and the $(8 ; 21)$ translocation protein (AML-1/ETO) as sequence-specific DNA-binding proteins: the runt homology domain is required for DNA binding and protein-protein interactions. Mol. Cell Biol. 13, 6336-6345 (1993)

48. Blount, A. L. et al. FoxL2 and Smad3 coordinately regulate follistatin gene transcription. J. Biol. Chem. 284, 7631-7645 (2009).

49. Barrionuevo, F. et al. Homozygous inactivation of Sox 9 causes complete XY sex reversal in mice. Biol. Reprod. 74, 195-201 (2006).

50. Wildey, G. M. \& Howe, P. H. Runx1 is a co-activator with FOXO3 to mediate transforming growth factor beta (TGFbeta)-induced Bim transcription in hepatic cells. J. Biol. Chem. 284, $20227-20239$ (2009).

51. Wang, L., Brugge, J. S. \& Janes, K. A. Intersection of FOXO- and RUNX1 mediated gene expression programs in single breast epithelial cells during morphogenesis and tumor progression. Proc. Natl Acad. Sci. USA 108, E803-E812 (2011).

52. Ono, M. et al. Foxp3 controls regulatory T-cell function by interacting with AML1/Runx1. Nature 446, 685-689 (2007).

53. Recouvreux, M. S. et al. RUNX1 and FOXP3 interplay regulates expression of breast cancer related genes. Oncotarget 7, 6552-6565 (2016).

54. Lichtinger, M. et al. RUNX1 reshapes the epigenetic landscape at the onset of haematopoiesis. EMBO J. 31, 4318-4333 (2012).

55. Munger, S. C., Natarajan, A., Looger, L. L., Ohler, U. \& Capel, B. Fine time course expression analysis identifies cascades of activation and repression and 
maps a putative regulator of mammalian sex determination. PLoS Genet. 9, e1003630 (2013).

56. Stevant, I. et al. Deciphering cell lineage specification during male sex determination with single-cell RNA sequencing. Cell Rep. 22, 1589-1599 (2018).

57. Nottingham, W. T. et al. Runx1-mediated hematopoietic stem-cell emergence is controlled by a Gata/Ets/SCL-regulated enhancer. Blood 110, 4188-4197 (2007).

58. Albrecht, K. H. \& Eicher, E. M. Evidence that Sry is expressed in pre-Sertoli cells and Sertoli and granulosa cells have a common precursor. Dev. Biol. 240, 92-107 (2001).

59. Rahmoun, M. et al. In mammalian foetal testes, SOX9 regulates expression of its target genes by binding to genomic regions with conserved signatures. Nucleic Acids Res. 45, 7191-7211 (2017).

60. Levasseur, A., Paquet, M., Boerboom, D. \& Boyer, A. Yes-associated protein and WW-containing transcription regulator 1 regulate the expression of sexdetermining genes in Sertoli cells, but their inactivation does not cause sex reversal. Biol. Reprod. 97, 162-175 (2017).

61. Uda, M. et al. Foxl2 disruption causes mouse ovarian failure by pervasive blockage of follicle development. Hum. Mol. Genet. 13, 1171-1181 (2004).

62. Nicol, B. \& Yao, H. H. Gonadal identity in the absence of pro-testis factor SOX9 and pro-ovary factor beta-catenin in mice. Biol. Reprod. 93, 35 (2015).

63. Marivin, E. et al. Sex hormone-binding globulins characterization and gonadal gene expression during sex differentiation in the rainbow trout, Oncorhynchus mykiss. Mol. Reprod. Dev. 81, 757-765 (2014).

64. Czerwinski, M., Natarajan, A., Barske, L., Looger, L. L. \& Capel, B. A timecourse analysis of systemic and gonadal effects of temperature on sexual development of the red-eared slider turtle Trachemys scripta elegans. Dev. Biol. 420, 166-177 (2016).

65. Elzaiat, M. et al. High-throughput sequencing analyses of XX genital ridges lacking FOXL2 reveal DMRT1 up-regulation before SOX9 expression during the sex-reversal process in goats. Biol. Reprod. 91, 153 (2014).

66. Umansky, K. B. et al. Runx1 transcription factor is required for myoblasts proliferation during muscle regeneration. PLoS Genet. 11, e1005457 (2015).

67. Heinz, S. et al. Simple combinations of lineage-determining transcription factors prime cis-regulatory elements required for macrophage and B cell identities. Mol. Cell 38, 576-589 (2010).

68. Chen, E. Y. et al. Enrichr: interactive and collaborative HTML5 gene list enrichment analysis tool. BMC Bioinformatics 14, 128 (2013).

69. Darde T. A., et al. The ReproGenomics Viewer: a multi-omics and crossspecies resource compatible with single-cell studies for the reproductive science community. Bioinformatics 31, 3133-3139 (2019).

70. Darde, T. A. et al. The ReproGenomics Viewer: an integrative cross-species toolbox for the reproductive science community. Nucleic Acids Res. 43, W109-W116 (2015).

\section{Acknowledgements}

We thank late Keith Parker (UT Southwestern Medical Center, USA) for the Sf1-Cre mice, David Schlessinger (National Institute on Aging, USA) for the Foxl2 $2^{+/}$mice, Yoram Groner and Ditsa Levanon (The Weizmann Institute of Science, Israel) for the RUNX1 antibody for ChIP, Susan Brenner-Morton and late Thomas Jessell (Columbia University, USA) for the RUNX1 antibody for immunofluorescences, David Zarkower (University of Minnesota, USA) for the DMRT1 antibody, Ken Morohashi (Kyushu
University, Japan) for the SF1 and SOX9 antibodies, and Dagmar Wilhelm (University of Melbourne, Australia) for the SRY antibody. We are grateful to the NIEHS Molecular Genomics Core, Integrative Bioinformatics Support Group, Comparative Medicine Branch, and Cellular and Molecular Pathology Branch for their services. This work was supported in part by the Intramural Research Program (ES102965) of the NIH, National Institute of Environmental Health Sciences in the U.S. The goat study was supported by Agence Nationale de la Recherche in France (ANR-16-CE14-0020). The human fetal gonads study was supported by l'Institut national de la santé et de la recherche médicale (Inserm), l'Université de Rennes 1 and l'Ecole des hautes études en santé publique (EHESP) in France. The study of red-eared slider turtle was supported by a grant (IOS1256675) from National Science Foundation in the US.

\section{Authors contributions}

B.N. performed the experiments in the mouse. B.N. and H.H.-C.Y. designed the study, analyzed data, and wrote the paper. S.A.G performed bioinformatic analyses. F.C and E.L. analyzed RUNX1 expression in human fetal gonads. M.P. and E.P. analyzed RUNX1 expression in the goat. E.D.-D.-P. and Y.G. analyzed runxl expression in rainbow trout. B.C. analyzed Runxl expression in the red-eared slider turtle. S.A.G., E.L., F.C., M.P., E.P., E.D.-D.-P., Y.G., B.C. and H.H.-C.Y. edited the paper.

\section{Competing interests}

The authors declare no competing interests.

\section{Additional information}

Supplementary information is available for this paper at https://doi.org/10.1038/s41467 019-13060-1.

Correspondence and requests for materials should be addressed to H.-C.Y.

Peer review information Nature Communications thanks the anonymous reviewer(s) for their contribution to the peer review of this work. Peer reviewer reports are available.

Reprints and permission information is available at http://www.nature.com/reprints

Publisher's note Springer Nature remains neutral with regard to jurisdictional claims in published maps and institutional affiliations.

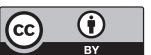

Open Access This article is licensed under a Creative Commons Attribution 4.0 International License, which permits use, sharing, adaptation, distribution and reproduction in any medium or format, as long as you give appropriate credit to the original author(s) and the source, provide a link to the Creative Commons license, and indicate if changes were made. The images or other third party material in this article are included in the article's Creative Commons license, unless indicated otherwise in a credit line to the material. If material is not included in the article's Creative Commons license and your intended use is not permitted by statutory regulation or exceeds the permitted use, you will need to obtain permission directly from the copyright holder. To view a copy of this license, visit http://creativecommons.org/ licenses/by/4.0/.

This is a U.S. Government work and not under copyright protection in the US; foreign copyright protection may apply 2019 